JOURNAL OF THE

AMERICAN MATHEMATICAL SOCIETY

Volume 21, Number 4, October 2008, Pages 951-979

S 0894-0347(07)00575-9

Article electronically published on June 14, 2007

\title{
BLOW-UP PHENOMENA FOR THE YAMABE EQUATION
}

\author{
SIMON BRENDLE
}

\section{INTRODUCTION}

Let $(M, g)$ be a compact Riemannian manifold of dimension $n \geq 3$. The Yamabe problem is concerned with finding metrics of constant scalar curvature in the conformal class of $g$. This problem can be reduced to a semi-linear elliptic PDE. Indeed, the metric $u^{\frac{4}{n-2}} g$ has constant scalar curvature $c$ if and only if

$$
\frac{4(n-1)}{n-2} \Delta_{g} u-R_{g} u+c u^{\frac{n+2}{n-2}}=0,
$$

where $\Delta_{g}$ is the Laplace operator with respect to $g$ and $R_{g}$ denotes the scalar curvature of $g$. Clearly, every solution of (11) is a critical point of the functional

$$
E_{g}(u)=\frac{\int_{M}\left(\frac{4(n-1)}{n-2}|d u|_{g}^{2}+R_{g} u^{2}\right) d v o l_{g}}{\left(\int_{M} u^{\frac{2 n}{n-2}} d v o l_{g}\right)^{\frac{n-2}{n}}} .
$$

It is well known that the PDE (1) has at least one positive solution for any choice of $(M, g)$. If $n \geq 6$ and $(M, g)$ is not locally conformally flat, this follows from results of T. Aubin [3]. The remaining cases were solved by R. Schoen using the positive mass theorem [16].

Solutions to (11) are not usually unique. As an example, consider the product metric on $S^{1}(L) \times S^{n-1}(1)$. If $L$ is sufficiently small, then the Yamabe PDE has a unique solution. On the other hand, there are many non-minimizing solutions if $L$ is large. D. Pollack 14 has used gluing techniques to construct high energy solutions on more general background manifolds: given any conformal class with positive Yamabe constant and any positive integer $N$, there exists a new conformal class which is close to the original one in the $C^{0}$-norm and contains at least $N$ metrics of constant scalar curvature (see [14, Theorem 0.1).

It is an interesting question whether the set of all solutions to the Yamabe PDE is compact (in the $C^{2}$-topology, say). A well-known conjecture states that this should be true unless $(M, g)$ is conformally equivalent to the round sphere (see [17, [18, [19]). This conjecture has been verified in low dimensions and in the locally conformally flat case: if $(M, g)$ is locally conformally flat, compactness follows from work of R. Schoen [17, 18. Moreover, Schoen proposed a strategy for proving the conjecture in the non-locally conformally flat case based on the Pohozaev identity.

Received by the editors October 23, 2006.

2000 Mathematics Subject Classification. Primary 53C21; Secondary 53C44.

Key words and phrases. Scalar curvature, conformal deformation of Riemannian metrics, blowup analysis.

This project was supported by the Alfred P. Sloan Foundation and by the National Science Foundation under grant DMS-0605223. 
In [12, Y.Y. Li and M. Zhu 12 followed this strategy to prove compactness in dimension 3. O. Druet [7] proved the conjecture in dimensions 4 and 5. Recently, F. Marques [13] showed that compactness holds up to dimension 7. The same result was obtained independently by Y.Y. Li and L. Zhang [11. Moreover, Li and Zhang showed that compactness holds in all dimensions provided that $\left|W_{g}(p)\right|+$ $\left|\nabla W_{g}(p)\right|>0$ for all $p \in M$. M. Khuri, F. Marques, and R. Schoen [10] proved compactness up to dimension 24, assuming that the positive mass theorem holds 1

In this paper, we address the opposite question: is it possible to construct Riemannian manifolds $(M, g)$ such that the set of constant scalar curvature metrics in the conformal class of $g$ is non-compact? So far, the only known examples where compactness fails involve non-smooth background metrics. The first result in this direction was established by A. Ambrosetti and A. Malchiodi [2]. This result was subsequently improved by M. Berti and A. Malchiodi [6]. Given positive integers $n$ and $k$ such that $k \geq 2$ and $n \geq 4 k+3$, Berti and Malchiodi showed that there exists a Riemannian metric $g$ on $S^{n}$ (of class $C^{k}$ ) for which the set of solutions to the Yamabe PDE (1) fails to be compact (see [6], Theorem 1.1). A survey of these results can be found in [1]. Recently, O. Druet and E. Hebey 8$]$ showed that blow-up can occur for problems of the form $L u+c u^{\frac{n+2}{n-2}}=0$, where $L$ is a lower order perturbation of the conformal Laplacian on $S^{n}$.

We improve the results of Berti and Malchiodi by showing that the set of solutions to the Yamabe PDE (1) can fail to be compact even if the background metric $g$ is $C^{\infty}$ smooth. In the examples we construct, the blowing-up sequence develops a singularity consisting of exactly one bubble.

Theorem. Assume that $n \geq 52$. Then there exists a Riemannian metric $g$ on $S^{n}$ (of class $C^{\infty}$ ) and a sequence of positive functions $v_{\nu} \in C^{\infty}\left(S^{n}\right)(\nu \in \mathbb{N})$ with the following properties:

(i) $g$ is not conformally flat,

(ii) $v_{\nu}$ is a solution of the Yamabe PDE (11) for all $\nu \in \mathbb{N}$,

(iii) $E_{g}\left(v_{\nu}\right)<Y\left(S^{n}\right)$ for all $\nu \in \mathbb{N}$, and $E_{g}\left(v_{\nu}\right) \rightarrow Y\left(S^{n}\right)$ as $\nu \rightarrow \infty$,

(iv) $\sup _{S^{n}} v_{\nu} \rightarrow \infty$ as $\nu \rightarrow \infty$.

(Here, $Y\left(S^{n}\right)$ denotes the Yamabe energy of the round metric on $S^{n}$.)

Let us sketch the main steps involved in the proof of the Theorem. For convenience, we will work on $\mathbb{R}^{n}$ instead of $S^{n}$. Let $g$ be a smooth metric on $\mathbb{R}^{n}$ which agrees with the Euclidean metric outside a ball of radius 1. We will assume throughout the paper that $\operatorname{det} g(x)=1$ for all $x \in \mathbb{R}^{n}$, so that the volume form associated with $g$ agrees with the Euclidean volume form.

Our goal is to construct solutions to the Yamabe PDE on $\left(\mathbb{R}^{n}, g\right)$. In Section 2 , we show that this problem can be reduced to finding critical points of a certain function $\mathcal{F}_{g}(\xi, \varepsilon)$, where $\xi$ is a vector in $\mathbb{R}^{n}$ and $\varepsilon$ is a positive real number. This idea has been used by many authors (see, e.g., 2 or 6). In Section 3 , we show that the function $\mathcal{F}_{g}(\xi, \varepsilon)$ can be approximated by an auxiliary function $F(\xi, \varepsilon)$. In Section 4 , we prove that the function $F(\xi, \varepsilon)$ has a critical point, which is a strict local minimum. Finally, in Section 5, we use a perturbation argument to construct critical points of the function $\mathcal{F}_{g}(\xi, \varepsilon)$. From this the main result follows.

\footnotetext{
${ }^{1} \mathrm{~T}$. Aubin has recently claimed a general compactness theorem in all dimensions 4], [5]. We have, however, been unable to verify some of the arguments in [4.
} 


\section{LyAPUNOV-SCHMIDT REDUCTION}

Let

$$
\mathcal{E}=\left\{w \in L^{\frac{2 n}{n-2}}\left(\mathbb{R}^{n}\right) \cap W_{l o c}^{1,2}\left(\mathbb{R}^{n}\right): \int_{\mathbb{R}^{n}}|d w|^{2}<\infty\right\} .
$$

By Sobolev's inequality, there exists a constant $K$, depending only on $n$, such that

$$
\left(\int_{\mathbb{R}^{n}}|w|^{\frac{2 n}{n-2}}\right)^{\frac{n-2}{n}} \leq K \int_{\mathbb{R}^{n}}|d w|^{2}
$$

for all $w \in \mathcal{E}$. We define a norm on $\mathcal{E}$ by $\|w\|_{\mathcal{E}}^{2}=\int_{\mathbb{R}^{n}}|d w|^{2}$. It is easy to see that $\mathcal{E}$, equipped with this norm, is complete.

Given any pair $(\xi, \varepsilon) \in \mathbb{R}^{n} \times(0, \infty)$, we define a function $u_{(\xi, \varepsilon)}: \mathbb{R}^{n} \rightarrow \mathbb{R}$ by

$$
u_{(\xi, \varepsilon)}(x)=\left(\frac{\varepsilon}{\varepsilon^{2}+|x-\xi|^{2}}\right)^{\frac{n-2}{2}} .
$$

The function $u_{(\xi, \varepsilon)}$ satisfies the elliptic PDE

$$
\Delta u_{(\xi, \varepsilon)}+n(n-2) u_{(\xi, \varepsilon)}^{\frac{n+2}{n-2}}=0 .
$$

It is well known that

$$
\int_{\mathbb{R}^{n}} u_{(\xi, \varepsilon)}^{\frac{2 n}{n-2}}=\left(\frac{Y\left(S^{n}\right)}{4 n(n-1)}\right)^{\frac{n}{2}}
$$

for all $(\xi, \varepsilon) \in \mathbb{R}^{n} \times(0, \infty)$. We next define

$$
\varphi_{(\xi, \varepsilon, 0)}(x)=\left(\frac{\varepsilon}{\varepsilon^{2}+|x-\xi|^{2}}\right)^{\frac{n+2}{2}} \frac{\varepsilon^{2}-|x-\xi|^{2}}{\varepsilon^{2}+|x-\xi|^{2}}
$$

and

$$
\varphi_{(\xi, \varepsilon, k)}(x)=\left(\frac{\varepsilon}{\varepsilon^{2}+|x-\xi|^{2}}\right)^{\frac{n+2}{2}} \frac{2 \varepsilon\left(x_{k}-\xi_{k}\right)}{\varepsilon^{2}+|x-\xi|^{2}}
$$

for $k=1, \ldots, n$. It is easy to see that the norm $\left\|\varphi_{(\xi, \varepsilon, k)}\right\|_{L^{\frac{2 n}{n+2}}\left(\mathbb{R}^{n}\right)}$ is constant in $\xi$ and $\varepsilon$. Finally, we define a closed subspace $\mathcal{E}_{(\xi, \varepsilon)} \subset \mathcal{E}$ by

$$
\mathcal{E}_{(\xi, \varepsilon)}=\left\{w \in \mathcal{E}: \int_{\mathbb{R}^{n}} \varphi_{(\xi, \varepsilon, k)} w=0 \quad \text { for } k=0,1, \ldots, n\right\} .
$$

Clearly, $u_{(\xi, \varepsilon)} \in \mathcal{E}_{(\xi, \varepsilon)}$.

Proposition 1. Consider a Riemannian metric on $\mathbb{R}^{n}$ of the form $g(x)=\exp (h(x))$, where $h(x)$ is a trace-free symmetric two-tensor on $\mathbb{R}^{n}$ satisfying $|h(x)|+|\partial h(x)|+$ $\left|\partial^{2} h(x)\right| \leq \alpha \leq 1$ for all $x \in \mathbb{R}^{n}$ and $h(x)=0$ for $|x| \geq 1$. There exists a constant $C$, depending only on $n$, such that

$$
\left\|\Delta_{g} u_{(\xi, \varepsilon)}-\frac{n-2}{4(n-1)} R_{g} u_{(\xi, \varepsilon)}+n(n-2) u_{(\xi, \varepsilon)}^{\frac{n+2}{n-2}}\right\|_{L^{\frac{2 n}{n+2}\left(\mathbb{R}^{n}\right)}} \leq C \alpha
$$

for all pairs $(\xi, \varepsilon) \in \mathbb{R}^{n} \times(0, \infty)$.

Proof. Using the pointwise estimate

$$
\begin{aligned}
& \left|\Delta_{g} u_{(\xi, \varepsilon)}-\frac{n-2}{4(n-1)} R_{g} u_{(\xi, \varepsilon)}+n(n-2) u_{(\xi, \varepsilon)}^{\frac{n+2}{n-2}}\right| \\
& \quad \leq C|h|\left|\partial^{2} u_{(\xi, \varepsilon)}\right|+C|\partial h|\left|\partial u_{(\xi, \varepsilon)}\right|+C\left(\left|\partial^{2} h\right|+|\partial h|^{2}\right) u_{(\xi, \varepsilon)},
\end{aligned}
$$


we obtain

$$
\begin{aligned}
& \left\|\Delta_{g} u_{(\xi, \varepsilon)}-\frac{n-2}{4(n-1)} R_{g} u_{(\xi, \varepsilon)}+n(n-2) u_{(\xi, \varepsilon)}^{\frac{n+2}{n-2}}\right\|_{L^{\frac{2 n}{n+2}}\left(\mathbb{R}^{n}\right)} \\
& \leq C\|h\|_{L^{\infty}\left(\mathbb{R}^{n}\right)}\left\|\partial^{2} u_{(\xi, \varepsilon)}\right\|_{L^{\frac{2 n}{n+2}}\left(\mathbb{R}^{n}\right)}+C\|\partial h\|_{L^{n}\left(\mathbb{R}^{n}\right)}\left\|\partial u_{(\xi, \varepsilon)}\right\|_{L^{2}\left(\mathbb{R}^{n}\right)} \\
& +C\left(\left\|\partial^{2} h\right\|_{L^{\frac{n}{2}\left(\mathbb{R}^{n}\right)}}+\|\partial h\|_{L^{n}\left(\mathbb{R}^{n}\right)}^{2}\right)\left\|u_{(\xi, \varepsilon)}\right\|_{L^{\frac{2 n}{n-2}}\left(\mathbb{R}^{n}\right)} \\
& \leq C \alpha .
\end{aligned}
$$

This proves the assertion.

Proposition 2. There exists a positive constant $\theta$, depending only on $n$, such that

$$
\begin{aligned}
& \int_{\mathbb{R}^{n}}\left(|d w|^{2}-n(n+2) u_{(\xi, \varepsilon)}^{\frac{4}{n-2}} w^{2}\right) \\
& \geq 2 \theta\|w\|_{\mathcal{E}}^{2}-\frac{16 n^{2}}{\theta}\left(\int_{\mathbb{R}^{n}} u_{(\xi, \varepsilon)}^{\frac{n+2}{n-2}} w\right)^{2}
\end{aligned}
$$

for all $w \in \mathcal{E}_{(\xi, \varepsilon)}$.

Proposition 2 follows from an analysis of the eigenvalues of the Laplace operator on $S^{n}$. The details can be found in [15].

Corollary 3. Consider a Riemannian metric on $\mathbb{R}^{n}$ of the form $g(x)=\exp (h(x))$, where $h(x)$ is a trace-free symmetric two-tensor on $\mathbb{R}^{n}$ satisfying $h(x)=0$ for $|x| \geq 1$. There exists a positive constant $\alpha_{0} \leq 1$, depending only on $n$, with the following property: if $|h(x)|+|\partial h(x)|+\left|\partial^{2} h(x)\right| \leq \alpha_{0}$ for all $x \in \mathbb{R}^{n}$, then we have

$$
\left(\int_{\mathbb{R}^{n}}|w|^{\frac{2 n}{n-2}}\right)^{\frac{n-2}{n}} \leq 2 K \int_{\mathbb{R}^{n}}\left(|d w|_{g}^{2}+\frac{n-2}{4(n-1)} R_{g} w^{2}\right)
$$

for all $w \in \mathcal{E}$ and

$$
\begin{aligned}
& \int_{\mathbb{R}^{n}}\left(|d w|_{g}^{2}+\frac{n-2}{4(n-1)} R_{g} w^{2}-n(n+2) u_{(\xi, \varepsilon)}^{\frac{4}{n-2}} w^{2}\right) \\
& \geq \theta\|w\|_{\mathcal{E}}^{2}-\frac{1}{\theta}\left(\int_{\mathbb{R}^{n}}\left(\Delta_{g} u_{(\xi, \varepsilon)}-\frac{n-2}{4(n-1)} R_{g} u_{(\xi, \varepsilon)}+n(n+2) u_{(\xi, \varepsilon)}^{\frac{n+2}{n-2}}\right) w\right)^{2}
\end{aligned}
$$

for all $w \in \mathcal{E}_{(\xi, \varepsilon)}$.

Proof. Using Proposition 1 and Hölder's inequality, we obtain

$$
\begin{aligned}
& \left|\int_{\mathbb{R}^{n}}\left(\Delta_{g} u_{(\xi, \varepsilon)}-\frac{n-2}{4(n-1)} R_{g} u_{(\xi, \varepsilon)}+n(n+2) u_{(\xi, \varepsilon)}^{\frac{n+2}{n-2}}\right) w\right| \\
& \geq 4 n\left|\int_{\mathbb{R}^{n}} u_{(\xi, \varepsilon)}^{\frac{n+2}{n-2}} w\right|-C \alpha_{0}\|w\|_{\mathcal{E}} .
\end{aligned}
$$

This implies

$$
\begin{aligned}
& \left(\int_{\mathbb{R}^{n}}\left(\Delta_{g} u_{(\xi, \varepsilon)}-\frac{n-2}{4(n-1)} R_{g} u_{(\xi, \varepsilon)}+n(n+2) u_{(\xi, \varepsilon)}^{\frac{n+2}{n-2}}\right) w\right)^{2} \\
& \geq 16 n^{2}\left(\int_{\mathbb{R}^{n}} u_{(\xi, \varepsilon)}^{\frac{n+2}{n-2}} w\right)^{2}-\theta^{2}\|w\|_{\mathcal{E}}^{2}
\end{aligned}
$$

if $\alpha_{0}$ is sufficiently small. Hence, the assertion follows from Proposition 2 , 
Proposition 4. Consider a Riemannian metric on $\mathbb{R}^{n}$ of the form $g(x)=\exp (h(x))$, where $h(x)$ is a trace-free symmetric two-tensor on $\mathbb{R}^{n}$ satisfying $|h(x)|+|\partial h(x)|+$ $\left|\partial^{2} h(x)\right| \leq \alpha_{0}$ for all $x \in \mathbb{R}^{n}$ and $h(x)=0$ for $|x| \geq 1$. Given any pair $(\xi, \varepsilon) \in \mathbb{R}^{n} \times$ $(0, \infty)$ and any function $f \in L^{\frac{2 n}{n+2}}\left(\mathbb{R}^{n}\right)$, there exists a unique function $w \in \mathcal{E}_{(\xi, \varepsilon)}$ such that

$$
\int_{\mathbb{R}^{n}}\left(\langle d w, d \psi\rangle_{g}+\frac{n-2}{4(n-1)} R_{g} w \psi-n(n+2) u_{(\xi, \varepsilon)}^{\frac{4}{n-2}} w \psi\right)=\int_{\mathbb{R}^{n}} f \psi
$$

for all test functions $\psi \in \mathcal{E}_{(\xi, \varepsilon)}$. Moreover, we have $\|w\|_{\mathcal{E}} \leq C\|f\|_{L^{\frac{2 n}{n+2}\left(\mathbb{R}^{n}\right)}}$, where $C$ is a constant that depends only on $n$.

Proof. Suppose that $w \in \mathcal{E}_{(\xi, \varepsilon)}$ and

$$
\int_{\mathbb{R}^{n}}\left(\langle d w, d \psi\rangle_{g}+\frac{n-2}{4(n-1)} R_{g} w \psi-n(n+2) u_{(\xi, \varepsilon)}^{\frac{4}{n-2}} w \psi\right)=\int_{\mathbb{R}^{n}} f \psi
$$

for all test functions $\psi \in \mathcal{E}_{(\xi, \varepsilon)}$. This implies

$$
\int_{\mathbb{R}^{n}}\left(|d w|_{g}^{2}+\frac{n-2}{4(n-1)} R_{g} w^{2}-n(n+2) u_{(\xi, \varepsilon)}^{\frac{4}{n-2}} w^{2}\right)=\int_{\mathbb{R}^{n}} f w
$$

and

$$
\int_{\mathbb{R}^{n}}\left(\Delta_{g} u_{(\xi, \varepsilon)}-\frac{n-2}{4(n-1)} R_{g} u_{(\xi, \varepsilon)}+n(n+2) u_{(\xi, \varepsilon)}^{\frac{n+2}{n-2}}\right) w=-\int_{\mathbb{R}^{n}} u_{(\xi, \varepsilon)} f .
$$

Using Corollary [3, we obtain

$$
\begin{aligned}
\theta\|w\|_{\mathcal{E}}^{2} & \leq \int_{\mathbb{R}^{n}}\left(|d w|_{g}^{2}+\frac{n-2}{4(n-1)} R_{g} w^{2}-n(n+2) u_{(\xi, \varepsilon)}^{\frac{4}{n-2}} w^{2}\right) \\
& +\frac{1}{\theta}\left(\int_{\mathbb{R}^{n}}\left(\Delta_{g} u_{(\xi, \varepsilon)}-\frac{n-2}{4(n-1)} R_{g} u_{(\xi, \varepsilon)}+n(n+2) u_{(\xi, \varepsilon)}^{\frac{n+2}{n-2}}\right) w\right)^{2} \\
& \leq\left(\int_{\mathbb{R}^{n}}|f|^{\frac{2 n}{n+2}}\right)^{\frac{n+2}{2 n}}\left(\int_{\mathbb{R}^{n}}|w|^{\frac{2 n}{n-2}}\right)^{\frac{n-2}{2 n}} \\
& +\frac{1}{\theta}\left(\int_{\mathbb{R}^{n}} u_{(\xi, \varepsilon)}^{\frac{2 n}{n-2}}\right)^{\frac{n-2}{n}}\left(\int_{\mathbb{R}^{n}}|f|^{\frac{2 n}{n+2}}\right)^{\frac{n+2}{n}} \\
& \leq K^{\frac{1}{2}}\|f\|_{L^{\frac{2 n}{n+2}\left(\mathbb{R}^{n}\right)}}\|w\|_{\mathcal{E}}+\frac{1}{\theta}\left(\frac{Y\left(S^{n}\right)}{4 n(n-1)}\right)^{\frac{n-2}{2}}\|f\|_{L^{\frac{2 n}{n+2}}\left(\mathbb{R}^{n}\right)}^{2} .
\end{aligned}
$$

Hence, it follows from Young's inequality that

$$
\frac{\theta}{2}\|w\|_{\mathcal{E}}^{2} \leq \frac{K}{2 \theta}\|f\|_{L^{\frac{2 n}{n+2}\left(\mathbb{R}^{n}\right)}}^{2}+\frac{1}{\theta}\left(\frac{Y\left(S^{n}\right)}{4 n(n-1)}\right)^{\frac{n-2}{2}}\|f\|_{L^{\frac{2 n}{n+2}}}^{2}\left(\mathbb{R}^{n}\right) .
$$

From this the uniqueness statement follows easily.

In order to prove the existence part, it suffices to minimize the functional

$$
\begin{aligned}
& \int_{\mathbb{R}^{n}}\left(|d w|_{g}^{2}+\frac{n-2}{4(n-1)} R_{g} w^{2}-n(n+2) u_{(\xi, \varepsilon)}^{\frac{4}{n-2}} w^{2}-2 f w\right) \\
& +\frac{1}{\theta}\left(\int_{\mathbb{R}^{n}}\left(\Delta_{g} u_{(\xi, \varepsilon)}-\frac{n-2}{4(n-1)} R_{g} u_{(\xi, \varepsilon)}+n(n+2) u_{(\xi, \varepsilon)}^{\frac{n+2}{n-2}}\right) w\right)^{2}
\end{aligned}
$$

over all functions $w \in \mathcal{E}_{(\xi, \varepsilon)}$. 
Proposition 5. Consider a Riemannian metric on $\mathbb{R}^{n}$ of the form $g(x)=\exp (h(x))$, where $h(x)$ is a trace-free symmetric two-tensor on $\mathbb{R}^{n}$ satisfying $h(x)=0$ for $|x| \geq 1$. Moreover, let $(\xi, \varepsilon) \in \mathbb{R}^{n} \times(0, \infty)$. There exists a positive constant $\alpha_{1} \leq \alpha_{0}$, depending only on $n$, with the following property: if $|h(x)|+|\partial h(x)|+\left|\partial^{2} h(x)\right| \leq \alpha_{1}$ for all $x \in \mathbb{R}^{n}$, then there exists a function $v_{(\xi, \varepsilon)} \in \mathcal{E}$ such that $v_{(\xi, \varepsilon)}-u_{(\xi, \varepsilon)} \in \mathcal{E}_{(\xi, \varepsilon)}$ and

$$
\int_{\mathbb{R}^{n}}\left(\left\langle d v_{(\xi, \varepsilon)}, d \psi\right\rangle_{g}+\frac{n-2}{4(n-1)} R_{g} v_{(\xi, \varepsilon)} \psi-n(n-2)\left|v_{(\xi, \varepsilon)}\right|^{\frac{4}{n-2}} v_{(\xi, \varepsilon)} \psi\right)=0
$$

for all test functions $\psi \in \mathcal{E}_{(\xi, \varepsilon)}$. Moreover, we have the estimate

$$
\begin{aligned}
& \left\|v_{(\xi, \varepsilon)}-u_{(\xi, \varepsilon)}\right\|_{\mathcal{E}} \\
& \leq C\left\|\Delta_{g} u_{(\xi, \varepsilon)}-\frac{n-2}{4(n-1)} R_{g} u_{(\xi, \varepsilon)}+n(n-2) u_{(\xi, \varepsilon)}^{\frac{n+2}{n-2}}\right\|_{L^{\frac{2 n}{n+2}}\left(\mathbb{R}^{n}\right)},
\end{aligned}
$$

where $C$ is a constant that depends only on $n$.

Proof. Let $G_{(\xi, \varepsilon)}: L^{\frac{2 n}{n+2}}\left(\mathbb{R}^{n}\right) \rightarrow \mathcal{E}_{(\xi, \varepsilon)}$ be the solution operator constructed in Proposition 4 We define a non-linear mapping $\Phi_{(\xi, \varepsilon)}: \mathcal{E}_{(\xi, \varepsilon)} \rightarrow \mathcal{E}_{(\xi, \varepsilon)}$ by

$$
\begin{aligned}
& \Phi_{(\xi, \varepsilon)}(w) \\
& =G_{(\xi, \varepsilon)}\left(\Delta_{g} u_{(\xi, \varepsilon)}-\frac{n-2}{4(n-1)} R_{g} u_{(\xi, \varepsilon)}+n(n-2) u_{(\xi, \varepsilon)}^{\frac{n+2}{n-2}}\right) \\
& +n(n-2) G_{(\xi, \varepsilon)}\left(\left|u_{(\xi, \varepsilon)}+w\right|^{\frac{4}{n-2}}\left(u_{(\xi, \varepsilon)}+w\right)-u_{(\xi, \varepsilon)}^{\frac{n+2}{n-2}}-\frac{n+2}{n-2} u_{(\xi, \varepsilon)}^{\frac{4}{n-2}} w\right) .
\end{aligned}
$$

It follows from Proposition 1 that $\left\|\Phi_{(\xi, \varepsilon)}(0)\right\|_{\mathcal{E}} \leq C \alpha_{1}$. Using the pointwise estimate

$$
\begin{aligned}
& || u_{(\xi, \varepsilon)}+\left.w\right|^{\frac{4}{n-2}}\left(u_{(\xi, \varepsilon)}+w\right)-\left|u_{(\xi, \varepsilon)}+\tilde{w}\right|^{\frac{4}{n-2}}\left(u_{(\xi, \varepsilon)}+\tilde{w}\right) \\
& \quad-\frac{n+2}{n-2} u_{(\xi, \varepsilon)}^{\frac{4}{n-2}}(w-\tilde{w}) \mid \\
& \leq C\left(|w|^{\frac{4}{n-2}}+|\tilde{w}|^{\frac{4}{n-2}}\right)|w-\tilde{w}|
\end{aligned}
$$

we obtain

$$
\begin{aligned}
& \left\|\Phi_{(\xi, \varepsilon)}(w)-\Phi_{(\xi, \varepsilon)}(\tilde{w})\right\|_{\mathcal{E}} \\
& \begin{aligned}
\leq C \| \mid u_{(\xi, \varepsilon)} & +\left.w\right|^{\frac{4}{n-2}}\left(u_{(\xi, \varepsilon)}+w\right)-\left|u_{(\xi, \varepsilon)}+\tilde{w}\right|^{\frac{4}{n-2}}\left(u_{(\xi, \varepsilon)}+\tilde{w}\right) \\
& -\frac{n+2}{n-2} u_{(\xi, \varepsilon)}^{\frac{4}{n-2}}(w-\tilde{w}) \|_{L^{\frac{2 n}{n+2}}\left(\mathbb{R}^{n}\right)} \\
\leq C\left(\|w\|_{L^{\frac{2 n}{n-2}}}^{\frac{4}{n-2}}\left(\mathbb{R}^{n}\right)\right. & \left.+\|\tilde{w}\|_{L^{\frac{2 n}{n-2}}\left(\mathbb{R}^{n}\right)}^{\frac{4}{n-2}}\right)\|w-\tilde{w}\|_{L^{\frac{2 n}{n-2}}\left(\mathbb{R}^{n}\right)}
\end{aligned}
\end{aligned}
$$

for all functions $w, \tilde{w} \in \mathcal{E}_{(\xi, \varepsilon)}$. This implies

$$
\left\|\Phi_{(\xi, \varepsilon)}(w)-\Phi_{(\xi, \varepsilon)}(\tilde{w})\right\|_{\mathcal{E}} \leq C\left(\|w\|_{\mathcal{E}}^{\frac{4}{n-2}}+\|\tilde{w}\|_{\mathcal{E}}^{\frac{4}{n-2}}\right)\|w-\tilde{w}\|_{\mathcal{E}}
$$

for $w, \tilde{w} \in \mathcal{E}_{(\xi, \varepsilon)}$. Hence, if $\alpha_{1}$ is sufficiently small, then the contraction mapping principle implies that the mapping $\Phi_{(\xi, \varepsilon)}$ has a unique fixed point. From this the assertion follows easily. 
We next define a function $\mathcal{F}_{g}: \mathbb{R}^{n} \times(0, \infty) \rightarrow \mathbb{R}$ by

$$
\begin{aligned}
\mathcal{F}_{g}(\xi, \varepsilon) & =\int_{\mathbb{R}^{n}}\left(\left|d v_{(\xi, \varepsilon)}\right|_{g}^{2}+\frac{n-2}{4(n-1)} R_{g} v_{(\xi, \varepsilon)}^{2}-(n-2)^{2}\left|v_{(\xi, \varepsilon)}\right|^{\frac{2 n}{n-2}}\right) \\
& -2(n-2)\left(\frac{Y\left(S^{n}\right)}{4 n(n-1)}\right)^{\frac{n}{2}} .
\end{aligned}
$$

If we choose $\alpha_{1}$ small enough, then we obtain the following result:

Proposition 6. The function $\mathcal{F}_{g}$ is continuously differentiable. Moreover, if $(\bar{\xi}, \bar{\varepsilon})$ is a critical point of the function $\mathcal{F}_{g}$, then the function $v_{(\bar{\xi}, \bar{\varepsilon})}$ is a non-negative weak solution of the equation

$$
\Delta_{g} v_{(\bar{\xi}, \bar{\varepsilon})}-\frac{n-2}{4(n-1)} R_{g} v_{(\bar{\xi}, \bar{\varepsilon})}+n(n-2) v_{(\bar{\xi}, \bar{\varepsilon})}^{\frac{n+2}{n-2}}=0 .
$$

Proof. By definition of $v_{(\xi, \varepsilon)}$, we can find real numbers $a_{k}(\xi, \varepsilon), k=0,1, \ldots, n$, such that

$$
\begin{aligned}
& \int_{\mathbb{R}^{n}}\left(\left\langle d v_{(\xi, \varepsilon)}, d \psi\right\rangle_{g}+\frac{n-2}{4(n-1)} R_{g} v_{(\xi, \varepsilon)} v_{(\xi, \varepsilon)} \psi-n(n-2)\left|v_{(\xi, \varepsilon)}\right|^{\frac{4}{n-2}} v_{(\xi, \varepsilon)} \psi\right) \\
& =\sum_{k=0}^{n} a_{k}(\xi, \varepsilon) \int_{\mathbb{R}^{n}} \varphi_{(\xi, \varepsilon, k)} \psi
\end{aligned}
$$

for all test functions $\psi \in \mathcal{E}$. This implies

$$
\frac{\partial}{\partial \varepsilon} \mathcal{F}_{g}(\varepsilon, \xi)=2 \sum_{k=0}^{n} a_{k}(\xi, \varepsilon) \int_{\mathbb{R}^{n}} \varphi_{\xi, \varepsilon, k)} \frac{\partial}{\partial \varepsilon} v_{(\xi, \varepsilon)}
$$

and

$$
\frac{\partial}{\partial \xi_{j}} \mathcal{F}_{g}(\varepsilon, \xi)=2 \sum_{k=0}^{n} a_{k}(\xi, \varepsilon) \int_{\mathbb{R}^{n}} \varphi_{(\xi, \varepsilon, k)} \frac{\partial}{\partial \xi_{j}} v_{(\xi, \varepsilon)}
$$

for $j=1, \ldots, n$. On the other hand, we have

$$
\int_{\mathbb{R}^{n}} \varphi_{(\xi, \varepsilon, k)}\left(v_{(\xi, \varepsilon)}-u_{(\xi, \varepsilon)}\right)=0
$$

since $v_{(\xi, \varepsilon)}-u_{(\xi, \varepsilon)} \in \mathcal{E}_{(\xi, \varepsilon)}$. This implies

$$
\begin{aligned}
0 & =\int_{\mathbb{R}^{n}} \frac{\partial}{\partial \varepsilon} \varphi_{(\xi, \varepsilon, k)}\left(v_{(\xi, \varepsilon)}-u_{(\xi, \varepsilon)}\right)+\int_{\mathbb{R}^{n}} \varphi_{(\xi, \varepsilon, k)} \frac{\partial}{\partial \varepsilon}\left(v_{(\xi, \varepsilon)}-u_{(\xi, \varepsilon)}\right) \\
& =\int_{\mathbb{R}^{n}} \frac{\partial}{\partial \varepsilon} \varphi_{(\xi, \varepsilon, k)}\left(v_{(\xi, \varepsilon)}-u_{(\xi, \varepsilon)}\right)+\int_{\mathbb{R}^{n}} \varphi_{(\xi, \varepsilon, k)} \frac{\partial}{\partial \varepsilon} v_{(\xi, \varepsilon)} \\
& +\frac{n-2}{2(n+1)}\left(\frac{Y\left(S^{n}\right)}{4 n(n-1)}\right)^{\frac{n}{2}} \varepsilon^{-1} \delta_{0 k}
\end{aligned}
$$

and

$$
\begin{aligned}
0 & =\int_{\mathbb{R}^{n}} \frac{\partial}{\partial \xi_{j}} \varphi_{(\xi, \varepsilon, k)}\left(v_{(\xi, \varepsilon)}-u_{(\xi, \varepsilon)}\right)+\int_{\mathbb{R}^{n}} \varphi_{(\xi, \varepsilon, k)} \frac{\partial}{\partial \xi_{j}}\left(v_{(\xi, \varepsilon)}-u_{(\xi, \varepsilon)}\right) \\
& =\int_{\mathbb{R}^{n}} \frac{\partial}{\partial \xi_{j}} \varphi_{(\xi, \varepsilon, k)}\left(v_{(\xi, \varepsilon)}-u_{(\xi, \varepsilon)}\right)+\int_{\mathbb{R}^{n}} \varphi_{(\xi, \varepsilon, k)} \frac{\partial}{\partial \xi_{j}} v_{(\xi, \varepsilon)} \\
& -\frac{n-2}{2(n+1)}\left(\frac{Y\left(S^{n}\right)}{4 n(n-1)}\right)^{\frac{n}{2}} \varepsilon^{-1} \delta_{j k}
\end{aligned}
$$


for $j=1, \ldots, n$. Putting these facts together, we obtain

$$
\begin{aligned}
& -\frac{n-2}{n+1}\left(\frac{Y\left(S^{n}\right)}{4 n(n-1)}\right)^{\frac{n}{2}} a_{0}(\xi, \varepsilon) \\
& =\varepsilon \frac{\partial}{\partial \varepsilon} \mathcal{F}_{g}(\xi, \varepsilon)+2 \varepsilon \sum_{k=0}^{n} a_{k}(\xi, \varepsilon) \int_{\mathbb{R}^{n}} \frac{\partial}{\partial \varepsilon} \varphi_{(\xi, \varepsilon, k)}\left(v_{(\xi, \varepsilon)}-u_{(\xi, \varepsilon)}\right)
\end{aligned}
$$

and

$$
\begin{aligned}
& \frac{n-2}{n+1}\left(\frac{Y\left(S^{n}\right)}{4 n(n-1)}\right)^{\frac{n}{2}} a_{j}(\xi, \varepsilon) \\
& =\varepsilon \frac{\partial}{\partial \xi_{j}} \mathcal{F}_{g}(\xi, \varepsilon)+2 \varepsilon \sum_{k=0}^{n} a_{k}(\xi, \varepsilon) \int_{\mathbb{R}^{n}} \frac{\partial}{\partial \xi_{j}} \varphi_{(\xi, \varepsilon, k)}\left(v_{(\xi, \varepsilon)}-u_{(\xi, \varepsilon)}\right)
\end{aligned}
$$

for $j=1, \ldots, n$. Hence, if $(\bar{\xi}, \bar{\varepsilon})$ is a critical point of $\mathcal{F}_{g}$, then we have

$$
\sum_{k=0}^{n}\left|a_{k}(\bar{\xi}, \bar{\varepsilon})\right| \leq C\left\|v_{(\bar{\xi}, \bar{\varepsilon})}-u_{(\bar{\xi}, \bar{\varepsilon})}\right\|_{L^{\frac{2 n}{n-2}}\left(\mathbb{R}^{n}\right)} \sum_{k=0}^{n}\left|a_{k}(\bar{\xi}, \bar{\varepsilon})\right|,
$$

where $C$ is a constant that depends only on $n$. On the other hand, we have

$$
\left\|v_{(\bar{\xi}, \bar{\varepsilon})}-u_{(\bar{\xi}, \bar{\varepsilon})}\right\|_{L^{\frac{2 n}{n-2}}\left(\mathbb{R}^{n}\right)} \leq C \alpha_{1} .
$$

Hence, if we choose $\alpha_{1}$ sufficiently small, then we must have $a_{k}(\bar{\xi}, \bar{\varepsilon})=0$ for $k=$ $0,1, \ldots, n$. Thus, we conclude that

$$
\int_{\mathbb{R}^{n}}\left(\left\langle d v_{(\bar{\xi}, \bar{\varepsilon})}, d \psi\right\rangle_{g}+\frac{n-2}{4(n-1)} R_{g} v_{(\bar{\xi}, \bar{\varepsilon})} \psi-n(n-2)\left|v_{(\bar{\xi}, \bar{\varepsilon})}\right|^{\frac{4}{n-2}} v_{(\bar{\xi}, \bar{\varepsilon})} \psi\right)=0
$$

for all test functions $\psi \in \mathcal{E}$. It remains to show that the function $v_{(\bar{\xi}, \bar{\varepsilon})}$ is nonnegative. To that end, we put $\psi=\min \left\{v_{(\bar{\xi}, \bar{\varepsilon})}, 0\right\}$. Since $v_{(\bar{\xi}, \bar{\varepsilon})} \in \mathcal{E}$, we conclude that $\psi \in \mathcal{E}$. This implies

$$
\begin{aligned}
& \int_{\left\{v_{(\bar{\xi}, \bar{\varepsilon})}<0\right\}}\left(\left|d v_{(\bar{\xi}, \bar{\varepsilon})}\right|_{g}^{2}+\frac{n-2}{4(n-1)} R_{g} v_{(\bar{\xi}, \bar{\varepsilon})}^{2}\right) \\
& =n(n-2) \int_{\left\{v_{(\bar{\xi}, \bar{\varepsilon})}<0\right\}}\left|v_{(\bar{\xi}, \bar{\varepsilon})}\right|^{\frac{2 n}{n-2}} .
\end{aligned}
$$

Moreover, we have

$$
\begin{aligned}
& \left(\int_{\left\{v_{(\bar{\xi}, \bar{\varepsilon})}<0\right\}}\left|v_{(\bar{\xi}, \bar{\varepsilon})}\right|^{\frac{2 n}{n-2}}\right)^{\frac{n-2}{n}} \\
& \leq 2 K \int_{\left\{v_{(\bar{\xi}, \bar{\varepsilon})}<0\right\}}\left(\left|d v_{(\bar{\xi}, \bar{\varepsilon})}\right|_{g}^{2}+\frac{n-2}{4(n-1)} R_{g} v_{(\bar{\xi}, \bar{\varepsilon})}^{2}\right)
\end{aligned}
$$

by Corollary 3 . From this we deduce that either $v_{(\bar{\xi}, \bar{\varepsilon})} \geq 0$ almost everywhere or

$$
\left(\int_{\left\{v_{(\bar{\xi}, \bar{\varepsilon})}<0\right\}}\left|v_{(\bar{\xi}, \bar{\varepsilon})}\right|^{\frac{2 n}{n-2}}\right)^{\frac{2}{n}} \geq \frac{1}{2 n(n-2) K} .
$$


On the other hand, we have

$$
\left(\int_{\left\{v_{(\bar{\xi}, \bar{\varepsilon})}<0\right\}}\left|v_{(\bar{\xi}, \bar{\varepsilon})}\right|^{\frac{2 n}{n-2}}\right)^{\frac{n-2}{2 n}} \leq\left(\int_{\mathbb{R}^{n}}\left|v_{(\bar{\xi}, \bar{\varepsilon})}-u_{(\bar{\xi}, \bar{\varepsilon})}\right|^{\frac{2 n}{n-2}}\right)^{\frac{n-2}{2 n}} \leq C \alpha_{1} .
$$

Hence, if $\alpha_{1}$ is sufficiently small, then we have $v_{(\bar{\xi}, \bar{\varepsilon})} \geq 0$ almost everywhere.

\section{An estimate for the energy of a "Bubble"}

Throughout this paper, we fix a multi-linear form $W: \mathbb{R}^{n} \times \mathbb{R}^{n} \times \mathbb{R}^{n} \times \mathbb{R}^{n} \rightarrow$ $\mathbb{R}$. We assume that $W_{i j k l}$ satisfy all the algebraic properties of the Weyl tensor. Moreover, we assume that some components of $W$ are non-zero, so that

$$
\sum_{i, j, k, l=1}^{n}\left(W_{i j k l}+W_{i l k j}\right)^{2}>0 .
$$

For abbreviation, we put

$$
H_{i k}(x)=\sum_{p, q=1}^{n} W_{i p k q} x_{p} x_{q}
$$

and

$$
\bar{H}_{i k}(x)=\left(1-|x|^{2}\right) H_{i k}(x) .
$$

It is easy to see that $H_{i k}(x)$ is trace-free, $\sum_{i=1}^{n} x_{i} H_{i k}(x)=0$, and $\sum_{i=1}^{n} \partial_{i} H_{i k}(x)=$ 0 for all $x \in \mathbb{R}^{n}$.

We consider a Riemannian metric of the form $g(x)=\exp (h(x))$, where $h(x)$ is a trace-free symmetric two-tensor on $\mathbb{R}^{n}$ satisfying $h(x)=0$ for $|x| \geq 1$,

$$
|h(x)|+|\partial h(x)|+\left|\partial^{2} h(x)\right| \leq \alpha_{1}
$$

for all $x \in \mathbb{R}^{n}$, and

$$
h_{i k}(x)=\mu\left(\lambda^{2}-|x|^{2}\right) H_{i k}(x)
$$

for $|x| \leq \rho$. We assume that the parameters $\lambda, \mu$, and $\rho$ are chosen such that $\mu \leq 1$ and $\lambda \leq \rho \leq 1$. Note that $\sum_{i=1}^{n} x_{i} h_{i k}(x)=0$ and $\sum_{i=1}^{n} \partial_{i} h_{i k}(x)=0$ for $|x| \leq \rho$.

Given any pair $(\xi, \varepsilon) \in \mathbb{R}^{n} \times(0, \infty)$, there exists a unique function $v_{(\xi, \varepsilon)}$ such that $v_{(\xi, \varepsilon)}-u_{(\xi, \varepsilon)} \in \mathcal{E}_{(\xi, \varepsilon)}$ and

$$
\int_{\mathbb{R}^{n}}\left(\left\langle d v_{(\xi, \varepsilon)}, d \psi\right\rangle_{g}+\frac{n-2}{4(n-1)} R_{g} v_{(\xi, \varepsilon)} \psi-n(n-2)\left|v_{(\xi, \varepsilon)}\right|^{\frac{4}{n-2}} v_{(\xi, \varepsilon)} \psi\right)=0
$$

for all test functions $\psi \in \mathcal{E}_{(\xi, \varepsilon)}$ (see Proposition 5). For abbreviation, let

$$
\Omega=\left\{(\xi, \varepsilon) \in \mathbb{R}^{n} \times \mathbb{R}:|\xi|<1, \frac{n-8}{3(n+4)}<\varepsilon^{2}<\frac{2(n-8)}{3(n+4)}\right\} .
$$

Proposition 7. For every pair $(\xi, \varepsilon) \in \lambda \Omega$, we have

$$
\begin{aligned}
& \left\|\Delta_{g} u_{(\xi, \varepsilon)}-\frac{n-2}{4(n-1)} R_{g} u_{(\xi, \varepsilon)}+n(n-2) u_{(\xi, \varepsilon)}^{\frac{n+2}{n-2}}\right\|_{L^{\frac{2 n}{n+2}}\left(\mathbb{R}^{n}\right)} \\
& \leq C \lambda^{4} \mu+C\left(\frac{\lambda}{\rho}\right)^{\frac{n-2}{2}}
\end{aligned}
$$


and

$$
\begin{aligned}
& \| \Delta_{g} u_{(\xi, \varepsilon)}-\frac{n-2}{4(n-1)} R_{g} u_{(\xi, \varepsilon)}+n(n-2) u_{(\xi, \varepsilon)}^{\frac{n+2}{n-2}} \\
& +\sum_{i, k=1}^{n} \mu\left(\lambda^{2}-|x|^{2}\right) H_{i k}(x) \partial_{i} \partial_{k} u_{(\xi, \varepsilon)} \|_{L^{\frac{2 n}{n+2}}\left(\mathbb{R}^{n}\right)} \\
& \leq C \lambda^{8} \mu^{2}+C\left(\frac{\lambda}{\rho}\right)^{\frac{n-2}{2}} .
\end{aligned}
$$

Proof. For abbreviation, we define two functions $A_{1}$ and $A_{2}$ by

$$
A_{1}=\Delta_{g} u_{(\xi, \varepsilon)}-\frac{n-2}{4(n-1)} R_{g} u_{(\xi, \varepsilon)}+n(n-2) u_{(\xi, \varepsilon)}^{\frac{n+2}{n-2}}
$$

and

$$
A_{2}=\sum_{i, k=1}^{n} \mu\left(\lambda^{2}-|x|^{2}\right) H_{i k}(x) \partial_{i} \partial_{k} u_{(\xi, \varepsilon)} .
$$

Using Proposition 26 and the identity $\sum_{i=1}^{n} \partial_{i} h_{i k}(x)=0$, we obtain

$$
\left|R_{g}(x)\right| \leq C|h(x)|^{2}\left|\partial^{2} h(x)\right|+C|\partial h(x)|^{2} \leq C \mu^{2}(\lambda+|x|)^{6}
$$

for $|x| \leq \rho$. This implies

$$
\begin{aligned}
\left|A_{1}\right| & =\left|\sum_{i, k=1}^{n} \partial_{i}\left[\left(g^{i k}-\delta_{i k}\right) \partial_{k} u_{(\xi, \varepsilon)}\right]-\frac{n-2}{4(n-1)} R_{g} u_{(\xi, \varepsilon)}\right| \\
& \leq C \lambda^{\frac{n-2}{2}} \mu(\lambda+|x|)^{4-n}
\end{aligned}
$$

and

$$
\begin{aligned}
\left|A_{1}+A_{2}\right| & =\left|\sum_{i, k=1}^{n} \partial_{i}\left[\left(g^{i k}-\delta_{i k}+h_{i k}\right) \partial_{k} u_{(\xi, \varepsilon)}\right]-\frac{n-2}{4(n-1)} R_{g} u_{(\xi, \varepsilon)}\right| \\
& \leq C \lambda^{\frac{n-2}{2}} \mu^{2}(\lambda+|x|)^{8-n}
\end{aligned}
$$

for $|x| \leq \rho$. Hence, we obtain

$$
\left\|A_{1}\right\|_{L^{\frac{2 n}{n+2}}\left(B_{\rho}(0)\right)} \leq C \lambda^{\frac{n-2}{2}} \mu\left(\int_{\mathbb{R}^{n}}(\lambda+|x|)^{-\frac{2 n(n-4)}{n+2}}\right)^{\frac{n+2}{2 n}} \leq C \lambda^{4} \mu
$$

and

$$
\left\|A_{1}+A_{2}\right\|_{L^{\frac{2 n}{n+2}}\left(B_{\rho}(0)\right)} \leq C \lambda^{\frac{n-2}{2}} \mu^{2}\left(\int_{\mathbb{R}^{n}}(\lambda+|x|)^{-\frac{2 n(n-8)}{n+2}}\right)^{\frac{n+2}{2 n}} \leq C \lambda^{8} \mu^{2} .
$$

On the other hand, we have

$$
\left|A_{1}(x)\right| \leq C \lambda^{\frac{n-2}{2}}|x|^{-n}
$$

for $\rho \leq|x| \leq 1$ and

$$
\left|A_{2}(x)\right| \leq C \lambda^{\frac{n-2}{2}} \mu|x|^{4-n}
$$

for $|x| \geq \rho$. Since the function $A_{1}(x)$ vanishes for $|x| \geq 1$, we conclude that

$$
\left\|A_{1}\right\|_{L^{\frac{2 n}{n+2}}\left(\mathbb{R}^{n} \backslash B_{\rho}(0)\right)} \leq C \lambda^{\frac{n-2}{2}}\left(\int_{\mathbb{R}^{n} \backslash B_{\rho}(0)}|x|^{-\frac{2 n^{2}}{n+2}}\right)^{\frac{n+2}{2 n}} \leq C\left(\frac{\lambda}{\rho}\right)^{\frac{n-2}{2}}
$$


and

$$
\left\|A_{2}\right\|_{L^{\frac{2 n}{n+2}\left(\mathbb{R}^{n} \backslash B_{\rho}(0)\right)}} \leq C \lambda^{\frac{n-2}{2}} \mu\left(\int_{\mathbb{R}^{n} \backslash B_{\rho}(0)}|x|^{-\frac{2 n(n-4)}{n+2}}\right)^{\frac{n+2}{2 n}} \leq C \rho^{4} \mu\left(\frac{\lambda}{\rho}\right)^{\frac{n-2}{2}} .
$$

Putting these facts together, the assertion follows.

Corollary 8. The function $v_{(\xi, \varepsilon)}-u_{(\xi, \varepsilon)}$ satisfies the estimate

$$
\left\|v_{(\xi, \varepsilon)}-u_{(\xi, \varepsilon)}\right\|_{L^{\frac{2 n}{n-2}}\left(\mathbb{R}^{n}\right)} \leq C \lambda^{4} \mu+C\left(\frac{\lambda}{\rho}\right)^{\frac{n-2}{2}}
$$

for $(\xi, \varepsilon) \in \lambda \Omega$.

Proof. It follows from Proposition [5 that

$$
\begin{aligned}
& \left\|v_{(\xi, \varepsilon)}-u_{(\xi, \varepsilon)}\right\|_{L^{\frac{2 n}{n-2}}\left(\mathbb{R}^{n}\right)} \\
& \leq C\left\|\Delta_{g} u_{(\xi, \varepsilon)}-\frac{n-2}{4(n-1)} R_{g} u_{(\xi, \varepsilon)}+n(n-2) u_{(\xi, \varepsilon)}^{\frac{n+2}{n-2}}\right\|_{L^{\frac{2 n}{n+2}}\left(\mathbb{R}^{n}\right)},
\end{aligned}
$$

where $C$ is a constant that depends only on $n$. Hence, the assertion follows from Proposition 7

We now prove a more refined estimate for the difference $v_{(\xi, \varepsilon)}-u_{(\xi, \varepsilon)}$. Using Proposition 4 with $h=0$, we conclude that there exists a unique function $w_{(\xi, \varepsilon)} \in$ $\mathcal{E}_{(\xi, \varepsilon)}$ such that

$$
\begin{aligned}
& \int_{\mathbb{R}^{n}}\left(\left\langle d w_{(\xi, \varepsilon)}, d \psi\right\rangle-n(n+2) u_{(\xi, \varepsilon)}^{\frac{4}{n-2}} w_{(\xi, \varepsilon)} \psi\right) \\
& =-\int_{\mathbb{R}^{n}} \sum_{i, k=1}^{n} \mu\left(\lambda^{2}-|x|^{2}\right) H_{i k}(x) \partial_{i} \partial_{k} u_{(\xi, \varepsilon)} \psi
\end{aligned}
$$

for all test functions $\psi \in \mathcal{E}_{(\xi, \varepsilon)}$.

Proposition 9. The function $w_{(\xi, \varepsilon)}$ is smooth. Moreover, if $(\xi, \varepsilon) \in \lambda \Omega$, then we have

$$
\begin{aligned}
& \left|w_{(\xi, \varepsilon)}(x)\right| \leq C \lambda^{\frac{n-2}{2}} \mu(\lambda+|x|)^{6-n}, \\
& \left|\partial w_{(\xi, \varepsilon)}(x)\right| \leq C \lambda^{\frac{n-2}{2}} \mu(\lambda+|x|)^{5-n}, \\
& \left|\partial^{2} w_{(\xi, \varepsilon)}(x)\right| \leq C \lambda^{\frac{n-2}{2}} \mu(\lambda+|x|)^{4-n}
\end{aligned}
$$

for all $x \in \mathbb{R}^{n}$.

Proof. Let $\varphi_{(\xi, \varepsilon, k)}$ be the functions defined in Section 2. We can find real numbers $b_{k}(\xi, \varepsilon), k=0,1, \ldots, n$, such that

$$
\begin{aligned}
& \int_{\mathbb{R}^{n}}\left(\left\langle d w_{(\xi, \varepsilon)}, d \psi\right\rangle-n(n+2) u_{(\xi, \varepsilon)}^{\frac{4}{n-2}} w_{(\xi, \varepsilon)} \psi\right) \\
& =-\int_{\mathbb{R}^{n}} \sum_{i, k=1}^{n} \mu\left(\lambda^{2}-|x|^{2}\right) H_{i k}(x) \partial_{i} \partial_{k} u_{(\xi, \varepsilon)} \psi+\sum_{k=0}^{n} b_{k}(\xi, \varepsilon) \int_{\mathbb{R}^{n}} \varphi_{(\xi, \varepsilon, k)} \psi
\end{aligned}
$$

for all test functions $\psi \in \mathcal{E}$. It follows from standard elliptic regularity theory that $w_{(\xi, \varepsilon)}$ is smooth. 
In the next step, we establish quantitative estimates for $w_{(\xi, \varepsilon)}$. To that end, we consider a pair $(\xi, \varepsilon) \in \lambda \Omega$. A straightforward calculation yields

$$
\left\|\sum_{i, k=1}^{n} \mu\left(\lambda^{2}-|x|^{2}\right) H_{i k}(x) \partial_{i} \partial_{k} u_{(\xi, \varepsilon)}\right\|_{L^{\frac{2 n}{n+2}}\left(\mathbb{R}^{n}\right)} \leq C \lambda^{4} \mu .
$$

From this we deduce that $\left\|w_{(\xi, \varepsilon)}\right\|_{L^{\frac{2 n}{n-2}}\left(\mathbb{R}^{n}\right)} \leq C \lambda^{4} \mu$ and $\sum_{k=0}^{n}\left|b_{k}(\xi, \varepsilon)\right| \leq C \lambda^{4} \mu$. This implies

$$
\begin{aligned}
& \left|\Delta w_{(\xi, \varepsilon)}+n(n+2) u_{(\xi, \varepsilon)}^{\frac{4}{n-2}} w_{(\xi, \varepsilon)}\right| \\
& =\left|\sum_{i, k=1}^{n} \mu\left(\lambda^{2}-|x|^{2}\right) H_{i k}(x) \partial_{i} \partial_{k} u_{(\xi, \varepsilon)}-\sum_{k=0}^{n} b_{k}(\xi, \varepsilon) \varphi_{(\xi, \varepsilon, k)}\right| \\
& \leq C \lambda^{\frac{n-2}{2}} \mu(\lambda+|x|)^{4-n}
\end{aligned}
$$

for all $x \in \mathbb{R}^{n}$. We claim that

$$
\sup _{x \in \mathbb{R}^{n}}(\lambda+|x|)^{\frac{n-2}{2}}\left|w_{(\xi, \varepsilon)}(x)\right| \leq C \lambda^{4} \mu .
$$

To show this, we fix a point $x_{0} \in \mathbb{R}^{n}$ and put $r=\frac{1}{2}\left(\lambda+\left|x_{0}\right|\right)$. Clearly, $\lambda+|x| \geq r$ for all $x \in B_{r}\left(x_{0}\right)$. This implies

$$
u_{(\xi, \varepsilon)}(x)^{\frac{4}{n-2}} \leq C r^{-2}
$$

and

$$
\left|\Delta w_{(\xi, \varepsilon)}+n(n+2) u_{(\xi, \varepsilon)}^{\frac{4}{n-2}} w_{(\xi, \varepsilon)}\right| \leq C \lambda^{\frac{n-2}{2}} \mu r^{4-n}
$$

for all $x \in B_{r}\left(x_{0}\right)$. Using standard interior estimates, we obtain

$$
\begin{aligned}
r^{\frac{n-2}{2}}\left|w_{(\xi, \varepsilon)}\left(x_{0}\right)\right| & \leq C\left\|w_{(\xi, \varepsilon)}\right\|_{L^{\frac{2 n}{n-2}}\left(B_{r}\left(x_{0}\right)\right)} \\
& +C r^{\frac{n+2}{2}}\left\|\Delta w_{(\xi, \varepsilon)}+n(n+2) u_{(\xi, \varepsilon)}^{\frac{4}{n-2}} w_{(\xi, \varepsilon)}\right\|_{L^{\infty}\left(B_{r}\left(x_{0}\right)\right)} \\
& \leq C \lambda^{4} \mu+C \lambda^{\frac{n-2}{2}} \mu r^{-\frac{n-10}{2}} \\
& \leq C \lambda^{4} \mu .
\end{aligned}
$$

Thus, we conclude that

$$
\sup _{x \in \mathbb{R}^{n}}(\lambda+|x|)^{\frac{n-2}{2}}\left|w_{(\xi, \varepsilon)}(x)\right| \leq C \lambda^{4} \mu,
$$

as claimed. Since $\sup _{x \in \mathbb{R}^{n}}|x|^{\frac{n-2}{2}}\left|w_{(\xi, \varepsilon)}(x)\right|<\infty$, we can express the function $w_{(\xi, \varepsilon)}$ in the form

$$
w_{(\xi, \varepsilon)}(x)=-\frac{1}{(n-2)\left|S^{n-1}\right|} \int_{\mathbb{R}^{n}}|x-y|^{2-n} \Delta w_{(\xi, \varepsilon)}(y) d y
$$

for all $x \in \mathbb{R}^{n}$.

We can now use a bootstrap argument to prove the desired estimate for $w_{(\xi, \varepsilon)}$. It follows from (5) that

$$
\sup _{x \in \mathbb{R}^{n}}(\lambda+|x|)^{\beta}\left|w_{(\xi, \varepsilon)}(x)\right| \leq C \sup _{x \in \mathbb{R}^{n}}(\lambda+|x|)^{\beta+2}\left|\Delta w_{(\xi, \varepsilon)}(x)\right|
$$


for all $0<\beta<n-2$. Since

$$
\begin{aligned}
\left|\Delta w_{(\xi, \varepsilon)}(x)\right| & \leq n(n+2) u_{(\xi, \varepsilon)}(x)^{\frac{4}{n-2}}\left|w_{(\xi, \varepsilon)}(x)\right| \\
& +C \lambda^{\frac{n-2}{2}} \mu(\lambda+|x|)^{4-n}
\end{aligned}
$$

for all $x \in \mathbb{R}^{n}$, we conclude that

$$
\begin{aligned}
\sup _{x \in \mathbb{R}^{n}}(\lambda+|x|)^{\beta}\left|w_{(\xi, \varepsilon)}(x)\right| & \leq C \lambda^{2} \sup _{x \in \mathbb{R}^{n}}(\lambda+|x|)^{\beta-2}\left|w_{(\xi, \varepsilon)}(x)\right| \\
& +C \lambda^{\beta-\frac{n-10}{2}} \mu
\end{aligned}
$$

for all $0<\beta \leq n-6$. Iterating this inequality, we obtain

$$
\sup _{x \in \mathbb{R}^{n}}(\lambda+|x|)^{n-6}\left|w_{(\xi, \varepsilon)}(x)\right| \leq C \lambda^{\frac{n-2}{2}} \mu .
$$

The estimates for the first and second derivatives of $w_{(\xi, \varepsilon)}$ follow now from standard interior estimates.

Corollary 10. The function $v_{(\xi, \varepsilon)}-u_{(\xi, \varepsilon)}-w_{(\xi, \varepsilon)}$ satisfies the estimate

$$
\left\|v_{(\xi, \varepsilon)}-u_{(\xi, \varepsilon)}-w_{(\xi, \varepsilon)}\right\|_{L^{\frac{2 n}{n-2}}\left(\mathbb{R}^{n}\right)} \leq C \lambda^{\frac{4(n+2)}{n-2}} \mu^{\frac{n+2}{n-2}}+C\left(\frac{\lambda}{\rho}\right)^{\frac{n-2}{2}}
$$

for $(\xi, \varepsilon) \in \lambda \Omega$.

Proof. Consider the functions

$$
B_{1}=\sum_{i, k=1}^{n} \partial_{i}\left[\left(g^{i k}-\delta_{i k}\right) \partial_{k} w_{(\xi, \varepsilon)}\right]-\frac{n-2}{4(n-1)} R_{g} w_{(\xi, \varepsilon)}
$$

and

$$
B_{2}=\sum_{i, k=1}^{n} \mu\left(\lambda^{2}-|x|^{2}\right) H_{i k}(x) \partial_{i} \partial_{k} u_{(\xi, \varepsilon)} .
$$

Using (3), we obtain

$$
\begin{aligned}
& \int_{\mathbb{R}^{n}}\left(\left\langle d w_{(\xi, \varepsilon)}, d \psi\right\rangle_{g}+\frac{n-2}{4(n-1)} R_{g} w_{(\xi, \varepsilon)} \psi-n(n+2) u_{(\xi, \varepsilon)}^{\frac{4}{n-2}} w_{(\xi, \varepsilon)} \psi\right) \\
& =-\int_{\mathbb{R}^{n}}\left(B_{1}+B_{2}\right) \psi
\end{aligned}
$$

for all functions $\psi \in \mathcal{E}_{(\xi, \varepsilon)}$. Since $w_{(\xi, \varepsilon)} \in \mathcal{E}_{(\xi, \varepsilon)}$, it follows that

$$
w_{(\xi, \varepsilon)}=-G_{(\xi, \varepsilon)}\left(B_{1}+B_{2}\right) .
$$

Moreover, we have

$$
v_{(\xi, \varepsilon)}-u_{(\xi, \varepsilon)}=G_{(\xi, \varepsilon)}\left(B_{3}+n(n-2) B_{4}\right),
$$

where

$$
B_{3}=\Delta_{g} u_{(\xi, \varepsilon)}-\frac{n-2}{4(n-1)} R_{g} u_{(\xi, \varepsilon)}+n(n-2) u_{(\xi, \varepsilon)}^{\frac{n+2}{n-2}}
$$

and

$$
B_{4}=\left|v_{(\xi, \varepsilon)}\right|^{\frac{4}{n-2}} v_{(\xi, \varepsilon)}-u_{(\xi, \varepsilon)}^{\frac{n+2}{n-2}}-\frac{n+2}{n-2} u_{(\xi, \varepsilon)}^{\frac{4}{n-2}}\left(v_{(\xi, \varepsilon)}-u_{(\xi, \varepsilon)}\right)
$$

Thus, we conclude that

$$
v_{(\xi, \varepsilon)}-u_{(\xi, \varepsilon)}-w_{(\xi, \varepsilon)}=G_{(\xi, \varepsilon)}\left(B_{1}+B_{2}+B_{3}+n(n-2) B_{4}\right),
$$


where $G_{(\xi, \varepsilon)}: L^{\frac{2 n}{n+2}}\left(\mathbb{R}^{n}\right) \rightarrow \mathcal{E}_{(\xi, \varepsilon)}$ denotes the solution operator constructed in Proposition 4. In particular, we have

$$
\left\|v_{(\xi, \varepsilon)}-u_{(\xi, \varepsilon)}-w_{(\xi, \varepsilon)}\right\|_{L^{\frac{2 n}{n-2}}\left(\mathbb{R}^{n}\right)} \leq C\left\|B_{1}+B_{2}+B_{3}+n(n-2) B_{4}\right\|_{L^{\frac{2 n}{n+2}}\left(\mathbb{R}^{n}\right)}
$$

by Proposition 4, Using Proposition 9, we obtain

$$
\left|B_{1}(x)\right| \leq C \lambda^{\frac{n-2}{2}} \mu^{2}(\lambda+|x|)^{8-n}
$$

for $|x| \leq \rho$ and

$$
\left|B_{1}(x)\right| \leq C \lambda^{\frac{n-2}{2}} \mu|x|^{4-n}
$$

for $\rho \leq|x| \leq 1$. Since the function $B_{1}(x)$ vanishes for $|x| \geq 1$, we conclude that

$$
\left\|B_{1}\right\|_{L^{\frac{2 n}{n+2}}\left(\mathbb{R}^{n}\right)} \leq C \lambda^{8} \mu^{2}+C \rho^{4} \mu\left(\frac{\lambda}{\rho}\right)^{\frac{n-2}{2}} .
$$

Moreover, we have

$$
\left\|B_{2}+B_{3}\right\|_{L^{\frac{2 n}{n+2}\left(\mathbb{R}^{n}\right)}} \leq C \lambda^{8} \mu^{2}+C\left(\frac{\lambda}{\rho}\right)^{\frac{n-2}{2}}
$$

by Proposition 7. Finally, the function $B_{4}$ satisfies a pointwise estimate of the form

$$
\left|B_{4}\right| \leq C\left|v_{(\xi, \varepsilon)}-u_{(\xi, \varepsilon)}\right|^{\frac{n+2}{n-2}}
$$

where $C$ is a constant that depends only on $n$. Hence, it follows from Corollary 8 that

$$
\begin{aligned}
\left\|B_{4}\right\|_{L^{\frac{2 n}{n+2}\left(\mathbb{R}^{n}\right)}} & \leq C\left\|v_{(\xi, \varepsilon)}-u_{(\xi, \varepsilon)}\right\|_{L^{\frac{2 n}{n-2}}\left(\mathbb{R}^{n}\right)}^{\frac{n+2}{n-2}} \\
& \leq C \lambda^{\frac{4(n+2)}{n-2}} \mu^{\frac{n+2}{n-2}}+C\left(\frac{\lambda}{\rho}\right)^{\frac{n+2}{2}} .
\end{aligned}
$$

Putting these facts together, we obtain

$$
\left\|v_{(\xi, \varepsilon)}-u_{(\xi, \varepsilon)}-w_{(\xi, \varepsilon)}\right\|_{L^{\frac{2 n}{n-2}}\left(\mathbb{R}^{n}\right)} \leq C \lambda^{\frac{4(n+2)}{n-2}} \mu^{\frac{n+2}{n-2}}+C\left(\frac{\lambda}{\rho}\right)^{\frac{n-2}{2}},
$$

as claimed.

Proposition 11. We have

$$
\begin{aligned}
\mid \int_{\mathbb{R}^{n}}\left(\left|d v_{(\xi, \varepsilon)}\right|_{g}^{2}-\left|d u_{(\xi, \varepsilon)}\right|_{g}^{2}+\frac{n-2}{4(n-1)} R_{g}\left(v_{(\xi, \varepsilon)}^{2}-u_{(\xi, \varepsilon)}^{2}\right)\right) \\
\quad+\int_{\mathbb{R}^{n}} n(n-2)\left(\left|v_{(\xi, \varepsilon)}\right|^{\frac{4}{n-2}}-u_{(\xi, \varepsilon)}^{\frac{4}{n-2}}\right) u_{(\xi, \varepsilon)} v_{(\xi, \varepsilon)} \\
\quad-\int_{\mathbb{R}^{n}} n(n-2)\left(\left|v_{(\xi, \varepsilon)}\right|^{\frac{2 n}{n-2}}-u_{(\xi, \varepsilon)}^{\frac{2 n}{n-2}}\right) \\
\quad-\int_{\mathbb{R}^{n}} \sum_{i, k=1}^{n} \mu\left(\lambda^{2}-|x|^{2}\right) H_{i k}(x) \partial_{i} \partial_{k} u_{(\xi, \varepsilon)} w_{(\xi, \varepsilon)} \mid \\
\leq C \lambda^{\frac{8 n}{n-2}} \mu^{\frac{2 n}{n-2}}+C \lambda^{4} \mu\left(\frac{\lambda}{\rho}\right)^{\frac{n-2}{2}}+C\left(\frac{\lambda}{\rho}\right)^{n-2}
\end{aligned}
$$

for $(\xi, \varepsilon) \in \lambda \Omega$. 
Proof. Using Proposition 5 with $\psi=v_{(\xi, \varepsilon)}-u_{(\xi, \varepsilon)}$, we obtain

$$
\begin{gathered}
\int_{\mathbb{R}^{n}}\left(\left|d v_{(\xi, \varepsilon)}\right|_{g}^{2}-\left\langle d u_{(\xi, \varepsilon)}, d v_{(\xi, \varepsilon)}\right\rangle_{g}+\frac{n-2}{4(n-1)} R_{g} v_{(\xi, \varepsilon)}\left(v_{(\xi, \varepsilon)}-u_{(\xi, \varepsilon)}\right)\right) \\
-\int_{\mathbb{R}^{n}} n(n-2)\left|v_{(\xi, \varepsilon)}\right|^{\frac{4}{n-2}} v_{(\xi, \varepsilon)}\left(v_{(\xi, \varepsilon)}-u_{(\xi, \varepsilon)}\right)=0 .
\end{gathered}
$$

Moreover, it follows from Proposition 7 and Corollary 8 that

$$
\begin{aligned}
& \mid \int_{\mathbb{R}^{n}}\left(\left\langle d u_{(\xi, \varepsilon)}, d v_{(\xi, \varepsilon)}\right\rangle_{g}-\left|d u_{(\xi, \varepsilon)}\right|_{g}^{2}+\frac{n-2}{4(n-1)} R_{g} u_{(\xi, \varepsilon)}\left(v_{(\xi, \varepsilon)}-u_{(\xi, \varepsilon)}\right)\right) \\
& \quad-\int_{\mathbb{R}^{n}} n(n-2) u_{(\xi, \varepsilon)}^{\frac{n+2}{n-2}}\left(v_{(\xi, \varepsilon)}-u_{(\xi, \varepsilon)}\right) \\
& \quad-\int_{\mathbb{R}^{n}} \sum_{i, k=1}^{n} \mu\left(\lambda^{2}-|x|^{2}\right) H_{i k}(x) \partial_{i} \partial_{k} u_{(\xi, \varepsilon)}\left(v_{(\xi, \varepsilon)}-u_{(\xi, \varepsilon)}\right) \mid \\
& \leq \| \Delta_{g} u_{(\xi, \varepsilon)}-\frac{n-2}{4(n-1)} R_{g} u_{(\xi, \varepsilon)}+n(n-2) u_{(\xi, \varepsilon)}^{\frac{n+2}{n-2}} \\
& \quad+\sum_{i, k=1}^{n} \mu\left(\lambda^{2}-|x|^{2}\right) H_{i k}(x) \partial_{i} \partial_{k} u_{(\xi, \varepsilon)} \|_{L^{\frac{2 n}{n+2}}\left(\mathbb{R}^{n}\right)} \\
& \quad \cdot\left\|v_{(\xi, \varepsilon)}-u_{(\xi, \varepsilon)}\right\|_{L^{\frac{2 n}{n-2}}\left(\mathbb{R}^{n}\right)} \\
& \leq C \lambda^{12} \mu^{3}+C \lambda^{4} \mu\left(\frac{\lambda}{\rho}\right)^{\frac{n-2}{2}}+C\left(\frac{\lambda}{\rho}\right)^{n-2} .
\end{aligned}
$$

Finally, we have

$$
\begin{aligned}
& \left|\int_{\mathbb{R}^{n}} \sum_{i, k=1}^{n} \mu\left(\lambda^{2}-|x|^{2}\right) H_{i k}(x) \partial_{i} \partial_{k} u_{(\xi, \varepsilon)}\left(v_{(\xi, \varepsilon)}-u_{(\xi, \varepsilon)}-w_{(\xi, \varepsilon)}\right)\right| \\
& \leq C \lambda^{4} \mu\left\|v_{(\xi, \varepsilon)}-u_{(\xi, \varepsilon)}-w_{(\xi, \varepsilon)}\right\|_{L^{\frac{2 n}{n-2}}\left(\mathbb{R}^{n}\right)} \\
& \leq C \lambda^{\frac{8 n}{n-2}} \mu^{\frac{2 n}{n-2}}+C \lambda^{4} \mu\left(\frac{\lambda}{\rho}\right)^{\frac{n-2}{2}}
\end{aligned}
$$

by (4) and Corollary 10. Putting these facts together, the assertion follows.

Proposition 12. We have

$$
\begin{aligned}
& \left|\int_{\mathbb{R}^{n}}\left(\left|v_{(\xi, \varepsilon)}\right|^{\frac{4}{n-2}}-u_{(\xi, \varepsilon)}^{\frac{4}{n-2}}\right) u_{(\xi, \varepsilon)} v_{(\xi, \varepsilon)}-\frac{2}{n} \int_{\mathbb{R}^{n}}\left(\left|v_{(\xi, \varepsilon)}\right|^{\frac{2 n}{n-2}}-u_{(\xi, \varepsilon)}^{\frac{2 n}{n-2}}\right)\right| \\
& \leq C \lambda^{\frac{8 n}{n-2}} \mu^{\frac{2 n}{n-2}}+C\left(\frac{\lambda}{\rho}\right)^{n}
\end{aligned}
$$

for $(\xi, \varepsilon) \in \lambda \Omega$.

Proof. We have the pointwise estimate

$$
\begin{aligned}
& \left|\left(\left|v_{(\xi, \varepsilon)}\right|^{\frac{4}{n-2}}-u_{(\xi, \varepsilon)}^{\frac{4}{n-2}}\right) u_{(\xi, \varepsilon)} v_{(\xi, \varepsilon)}-\frac{2}{n}\left(\left|v_{(\xi, \varepsilon)}\right|^{\frac{2 n}{n-2}}-u_{(\xi, \varepsilon)}^{\frac{2 n}{n-2}}\right)\right| \\
& \quad \leq C\left|v_{(\xi, \varepsilon)}-u_{(\xi, \varepsilon)}\right|^{\frac{2 n}{n-2}}
\end{aligned}
$$


where $C$ is a constant that depends only on $n$. This implies

$$
\begin{aligned}
& \left|\int_{\mathbb{R}^{n}}\left(\left|v_{(\xi, \varepsilon)}\right|^{\frac{4}{n-2}}-u_{(\xi, \varepsilon)}^{\frac{4}{n-2}}\right) u_{(\xi, \varepsilon)} v_{(\xi, \varepsilon)}-\frac{2}{n} \int_{\mathbb{R}^{n}}\left(\left|v_{(\xi, \varepsilon)}\right|^{\frac{2 n}{n-2}}-u_{(\xi, \varepsilon)}^{\frac{2 n}{n-2}}\right)\right| \\
& \leq C\left\|v_{(\xi, \varepsilon)}-u_{(\xi, \varepsilon)}\right\|_{L^{\frac{2 n}{n-2}}\left(\mathbb{R}^{n}\right)}^{\frac{2 n}{n-2}} \\
& \leq C \lambda^{\frac{8 n}{n-2}} \mu^{\frac{2 n}{n-2}}+C\left(\frac{\lambda}{\rho}\right)^{n}
\end{aligned}
$$

by Corollary 8 ,

Proposition 13. We have

$$
\begin{gathered}
\mid \int_{\mathbb{R}^{n}}\left(\left|d u_{(\xi, \varepsilon)}\right|_{g}^{2}+\frac{n-2}{4(n-1)} R_{g} u_{(\xi, \varepsilon)}^{2}-n(n-2) u_{(\xi, \varepsilon)}^{\frac{2 n}{n-2}}\right) \\
\quad-\int_{B_{\rho}(0)} \frac{1}{2} \sum_{i, k, l=1}^{n} h_{i l} h_{k l} \partial_{i} u_{(\xi, \varepsilon)} \partial_{k} u_{(\xi, \varepsilon)} \\
\quad+\int_{B_{\rho}(0)} \frac{n-2}{16(n-1)} \sum_{i, k, l=1}^{n}\left(\partial_{l} h_{i k}\right)^{2} u_{(\xi, \varepsilon)}^{2} \mid \\
\leq C \lambda^{12} \mu^{3}+C\left(\frac{\lambda}{\rho}\right)^{n-2}
\end{gathered}
$$

for all $(\xi, \varepsilon) \in \lambda \Omega$.

Proof. Note that

$$
\begin{aligned}
& \left|g^{i k}(x)-\delta_{i k}+h_{i k}(x)-\frac{1}{2} \sum_{l=1}^{n} h_{i l}(x) h_{k l}(x)\right| \\
& \leq C|h(x)|^{3} \leq C \mu^{3}(\lambda+|x|)^{12}
\end{aligned}
$$

for $|x| \leq \rho$. This implies

$$
\begin{aligned}
& \mid \int_{\mathbb{R}^{n}}\left(\left|d u_{(\xi, \varepsilon)}\right|_{g}^{2}-\left|d u_{(\xi, \varepsilon)}\right|^{2}\right)+\int_{\mathbb{R}^{n}} \sum_{i, k=1}^{n} h_{i k} \partial_{i} u_{(\xi, \varepsilon)} \partial_{k} u_{(\xi, \varepsilon)} \\
& \quad-\int_{B_{\rho}(0)} \frac{1}{2} \sum_{i, k, l=1}^{n} h_{i l} h_{k l} \partial_{i} u_{(\xi, \varepsilon)} \partial_{k} u_{(\xi, \varepsilon)} \mid \\
& \leq C \lambda^{n-2} \mu^{3} \int_{B_{\rho}(0)}(\lambda+|x|)^{14-2 n}+C \lambda^{n-2} \int_{\mathbb{R}^{n} \backslash B_{\rho}(0)}(\lambda+|x|)^{2-2 n} \\
& \leq C \lambda^{12} \mu^{3}+C\left(\frac{\lambda}{\rho}\right)^{n-2} .
\end{aligned}
$$

By Proposition 26, the scalar curvature of $g$ satisfies the estimate

$$
\begin{aligned}
& \left|R_{g}(x)+\frac{1}{4} \sum_{i, k, l=1}^{n}\left(\partial_{l} h_{i k}(x)\right)^{2}\right| \\
& \leq C|h(x)|^{2}\left|\partial^{2} h(x)\right|+C|h(x)||\partial h(x)|^{2} \\
& \leq C \mu^{3}(\lambda+|x|)^{10}
\end{aligned}
$$


for $|x| \leq \rho$. This implies

$$
\begin{aligned}
& \left|\int_{\mathbb{R}^{n}} R_{g} u_{(\xi, \varepsilon)}^{2}+\int_{B_{\rho}(0)} \frac{1}{4} \sum_{i, k, l=1}^{n}\left(\partial_{l} h_{i k}\right)^{2} u_{(\xi, \varepsilon)}^{2}\right| \\
& \leq C \lambda^{12} \mu^{3} \int_{B_{\rho}(0)}(\lambda+|x|)^{14-2 n}+C \lambda^{n-2} \int_{\mathbb{R}^{n} \backslash B_{\rho}(0)}(\lambda+|x|)^{4-2 n} \\
& \leq C \lambda^{12} \mu^{3}+C \rho^{2}\left(\frac{\lambda}{\rho}\right)^{n-2} .
\end{aligned}
$$

At this point, we use the formula

$$
\begin{aligned}
& \partial_{i} u_{(\xi, \varepsilon)} \partial_{k} u_{(\xi, \varepsilon)}-\frac{n-2}{4(n-1)} \partial_{i} \partial_{k}\left(u_{(\xi, \varepsilon)}^{2}\right) \\
& =\frac{1}{n}\left(\left|d u_{(\xi, \varepsilon)}\right|^{2}-\frac{n-2}{4(n-1)} \Delta\left(u_{(\xi, \varepsilon)}^{2}\right)\right) \delta_{i k} .
\end{aligned}
$$

Since $h_{i k}$ is trace-free, we obtain

$$
\sum_{i, k=1}^{n} h_{i k} \partial_{i} u_{(\xi, \varepsilon)} \partial_{k} u_{(\xi, \varepsilon)}=\frac{n-2}{4(n-1)} \sum_{i, k=1}^{n} h_{i k} \partial_{i} \partial_{k}\left(u_{(\xi, \varepsilon)}^{2}\right)
$$

hence

$$
\int_{\mathbb{R}^{n}} \sum_{i, k=1}^{n} h_{i k} \partial_{i} u_{(\xi, \varepsilon)} \partial_{k} u_{(\xi, \varepsilon)}=\int_{\mathbb{R}^{n}} \frac{n-2}{4(n-1)} \sum_{i, k=1}^{n} \partial_{i} \partial_{k} h_{i k} u_{(\xi, \varepsilon)}^{2} .
$$

Since $\sum_{i=1}^{n} \partial_{i} h_{i k}(x)=0$ for $|x| \leq \rho$, it follows that

$$
\left|\int_{\mathbb{R}^{n}} \sum_{i, k=1}^{n} h_{i k} \partial_{i} u_{(\xi, \varepsilon)} \partial_{k} u_{(\xi, \varepsilon)}\right| \leq C \int_{\mathbb{R}^{n} \backslash B_{\rho}(0)} u_{(\xi, \varepsilon)}^{2} \leq C \rho^{2}\left(\frac{\lambda}{\rho}\right)^{n-2} .
$$

Putting these facts together, the assertion follows.

Corollary 14. The function $\mathcal{F}_{g}(\xi, \varepsilon)$ satisfies the estimate

$$
\begin{aligned}
& \mid \mathcal{F}_{g}(\xi, \varepsilon)-\int_{B_{\rho}(0)} \frac{1}{2} \sum_{i, k, l=1}^{n} h_{i l} h_{k l} \partial_{i} u_{(\xi, \varepsilon)} \partial_{k} u_{(\xi, \varepsilon)} \\
&+\int_{B_{\rho}(0)} \frac{n-2}{16(n-1)} \sum_{i, k, l=1}^{n}\left(\partial_{l} h_{i k}\right)^{2} u_{(\xi, \varepsilon)}^{2} \\
&-\int_{\mathbb{R}^{n}} \sum_{i, k=1}^{n} \mu\left(\lambda^{2}-|x|^{2}\right) H_{i k}(x) \partial_{i} \partial_{k} u_{(\xi, \varepsilon)} w_{(\xi, \varepsilon)} \mid \\
& \leq C \lambda^{\frac{8 n}{n-2}} \mu^{\frac{2 n}{n-2}}+C \lambda^{4} \mu\left(\frac{\lambda}{\rho}\right)^{\frac{n-2}{2}}+C\left(\frac{\lambda}{\rho}\right)^{n-2}
\end{aligned}
$$

for $(\xi, \varepsilon) \in \lambda \Omega$.

Proof. This follows by combining Proposition 11, Proposition [12, and Proposition 13. 


\section{Finding A CRITICAL POINT OF AN AUXILIARY FUNCTION}

We define a function $F: \mathbb{R}^{n} \times(0, \infty) \rightarrow \mathbb{R}$ as follows: given any pair $(\xi, \varepsilon) \in$ $\mathbb{R}^{n} \times(0, \infty)$, we define

$$
\begin{aligned}
F(\xi, \varepsilon) & =\int_{\mathbb{R}^{n}} \frac{1}{2} \sum_{i, k, l=1}^{n} \bar{H}_{i l}(x) \bar{H}_{k l}(x) \partial_{i} u_{(\xi, \varepsilon)}(x) \partial_{k} u_{(\xi, \varepsilon)}(x) \\
& -\int_{\mathbb{R}^{n}} \frac{n-2}{16(n-1)} \sum_{i, k, l=1}^{n}\left(\partial_{l} \bar{H}_{i k}(x)\right)^{2} u_{(\xi, \varepsilon)}(x)^{2} \\
& +\int_{\mathbb{R}^{n}} \sum_{i, k=1}^{n} \bar{H}_{i k}(x) \partial_{i} \partial_{k} u_{(\xi, \varepsilon)}(x) z_{(\xi, \varepsilon)}(x),
\end{aligned}
$$

where $z_{(\xi, \varepsilon)} \in \mathcal{E}_{(\xi, \varepsilon)}$ satisfies the relation

$$
\begin{aligned}
& \int_{\mathbb{R}^{n}}\left(\left\langle d z_{(\xi, \varepsilon)}, d \psi\right\rangle-n(n+2) u_{(\xi, \varepsilon)}(x)^{\frac{4}{n-2}} z_{(\xi, \varepsilon)} \psi\right) \\
& =-\int_{\mathbb{R}^{n}} \sum_{i, k=1}^{n} \bar{H}_{i k} \partial_{i} \partial_{k} u_{(\xi, \varepsilon)} \psi
\end{aligned}
$$

for all test functions $\psi \in \mathcal{E}_{(\xi, \varepsilon)}$. Our goal in this section is to show that the function $F(\xi, \varepsilon)$ has a critical point.

Proposition 15. The function $F(\xi, \varepsilon)$ satisfies $F(\xi, \varepsilon)=F(-\xi, \varepsilon)$ for all $(\xi, \varepsilon) \in$ $\mathbb{R}^{n} \times(0, \infty)$. Consequently, we have $\frac{\partial}{\partial \xi_{p}} F(0, \varepsilon)=0$ and $\frac{\partial^{2}}{\partial \varepsilon \partial \xi_{p}} F(0, \varepsilon)=0$ for all $\varepsilon>0$ and $p=1, \ldots, n$.

Proof. This follows immediately from the relation $\bar{H}_{i k}(-x)=\bar{H}_{i k}(x)$.

Proposition 16. We have

$$
\begin{aligned}
& \int_{\partial B_{r}(0)} \sum_{i, k, l=1}^{n}\left(\partial_{l} H_{i k}(x)\right)^{2} x_{p} x_{q} \\
& =\frac{2}{n(n+2)}\left|S^{n-1}\right| \sum_{i, k, l=1}^{n}\left(W_{i p k l}+W_{i l k p}\right)\left(W_{i q k l}+W_{i l k q}\right) r^{n+3} \\
& +\frac{1}{n(n+2)}\left|S^{n-1}\right| \sum_{i, j, k, l=1}^{n}\left(W_{i j k l}+W_{i l k j}\right)^{2} \delta_{p q} r^{n+3}
\end{aligned}
$$

and

$$
\begin{aligned}
& \int_{\partial B_{r}(0)} \sum_{i, k=1}^{n} H_{i k}(x)^{2} x_{p} x_{q} \\
& =\frac{2}{n(n+2)(n+4)}\left|S^{n-1}\right| \sum_{i, k, l=1}^{n}\left(W_{i p k l}+W_{i l k p}\right)\left(W_{i q k l}+W_{i l k q}\right) r^{n+5} \\
& +\frac{1}{2 n(n+2)(n+4)}\left|S^{n-1}\right| \sum_{i, j, k, l=1}^{n}\left(W_{i j k l}+W_{i l k j}\right)^{2} \delta_{p q} r^{n+5} .
\end{aligned}
$$


Proof. By definition of $H_{i k}(x)$, we have

$$
\begin{aligned}
& \int_{\partial B_{r}(0)} \sum_{i, k, l=1}^{n}\left(\partial_{l} H_{i k}(x)\right)^{2} x_{p} x_{q} \\
& =\int_{\partial B_{r}(0)} \sum_{i, j, k, l, m=1}^{n}\left(W_{i j k l}+W_{i l k j}\right)\left(W_{i m k l}+W_{i l k m}\right) x_{j} x_{m} x_{p} x_{q} \\
& =\frac{2}{n(n+2)}\left|S^{n-1}\right| \sum_{i, k, l=1}^{n}\left(W_{i p k l}+W_{i l k p}\right)\left(W_{i q k l}+W_{i l k q}\right) r^{n+3} \\
& +\frac{1}{n(n+2)}\left|S^{n-1}\right| \sum_{i, j, k, l=1}^{n}\left(W_{i j k l}+W_{i l k j}\right)^{2} \delta_{p q} r^{n+3} .
\end{aligned}
$$

Moreover, it follows from Corollary 29 that

$$
\begin{aligned}
& \int_{\partial B_{r}(0)} \sum_{i, k=1}^{n} H_{i k}(x)^{2} x_{p} x_{q} \\
& =\int_{\partial B_{r}(0)} \sum_{i, j, k, l, m, s=1}^{n} W_{i j k l} W_{i m k s} x_{j} x_{l} x_{m} x_{s} x_{p} x_{q} \\
& =\frac{2}{n(n+2)(n+4)}\left|S^{n-1}\right| \sum_{i, k, l=1}^{n}\left(W_{i p k l}+W_{i l k p}\right)\left(W_{i q k l}+W_{i l k q}\right) r^{n+5} \\
& +\frac{1}{2 n(n+2)(n+4)}\left|S^{n-1}\right| \sum_{i, j, k, l=1}^{n}\left(W_{i j k l}+W_{i l k j}\right)^{2} \delta_{p q} r^{n+5} .
\end{aligned}
$$

This completes the proof.

Proposition 17. We have

$$
\begin{aligned}
& \int_{\partial B_{r}(0)} \sum_{i, k, l=1}^{n}\left(\partial_{l} \bar{H}_{i k}(x)\right)^{2} x_{p} x_{q} \\
& =\frac{2}{n(n+2)}\left|S^{n-1}\right| \sum_{i, k, l=1}^{n}\left(W_{i p k l}+W_{i l k p}\right)\left(W_{i q k l}+W_{i l k q}\right) \\
& \cdot\left[r^{n+3}-\frac{2(n+8)}{n+4} r^{n+5}+\frac{n+16}{n+4} r^{n+7}\right] \\
& +\frac{1}{n(n+2)}\left|S^{n-1}\right| \sum_{i, j, k, l=1}^{n}\left(W_{i j k l}+W_{i l k j}\right)^{2} \delta_{p q} \\
& \cdot\left[r^{n+3}-\frac{2(n+6)}{n+4} r^{n+5}+\frac{n+10}{n+4} r^{n+7}\right]
\end{aligned}
$$

Proof. Using the identity

$$
\partial_{l} \bar{H}_{i k}(x)=\left(1-|x|^{2}\right) \partial_{l} H_{i k}(x)-2 H_{i k}(x) x_{l}
$$


and Euler's theorem, we obtain

$$
\begin{aligned}
& \sum_{i, k, l=1}^{n}\left(\partial_{l} \bar{H}_{i k}(x)\right)^{2} \\
& =\left(1-|x|^{2}\right)^{2} \sum_{i, k, l=1}^{n}\left(\partial_{l} H_{i k}(x)\right)^{2} \\
& -4\left(1-|x|^{2}\right) \sum_{i, k, l=1}^{n} H_{i k}(x) x_{l} \partial_{l} H_{i k}(x)+4|x|^{2} \sum_{i, k=1}^{n} H_{i k}(x)^{2} \\
& =\left(1-|x|^{2}\right)^{2} \sum_{i, k, l=1}^{n}\left(\partial_{l} H_{i k}(x)\right)^{2}-4\left(2-3|x|^{2}\right) \sum_{i, k=1}^{n} H_{i k}(x)^{2} .
\end{aligned}
$$

Hence, the assertion follows from the previous proposition.

Corollary 18. We have

$$
\begin{aligned}
& \int_{\partial B_{r}(0)} \sum_{i, k, l=1}^{n}\left(\partial_{l} \bar{H}_{i k}(x)\right)^{2}=\frac{1}{n} \mid S^{n-1} \mid \sum_{i, j, k, l=1}^{n}\left(W_{i j k l}+W_{i l k j}\right)^{2} \\
& \cdot\left[r^{n+1}-\frac{2(n+4)}{n+2} r^{n+3}+\frac{n+8}{n+2} r^{n+5}\right] .
\end{aligned}
$$

Proposition 19. We have

$$
\begin{aligned}
F(0, \varepsilon)=-\frac{(n-2)(n+4)}{16 n(n-1)(n+2)}\left|S^{n-1}\right| \sum_{i, j, k, l=1}^{n}\left(W_{i j k l}+W_{i l k j}\right)^{2} \\
\cdot\left[\frac{n-8}{n+4} \varepsilon^{4}-2 \varepsilon^{6}+\frac{n+8}{n-10} \varepsilon^{8}\right] \int_{0}^{\infty}\left(1+r^{2}\right)^{2-n} r^{n+3} d r .
\end{aligned}
$$

Proof. Note that $z_{(0, \varepsilon)}(x)=0$ for all $x \in \mathbb{R}^{n}$. This implies

$$
F(0, \varepsilon)=-\int_{\mathbb{R}^{n}} \frac{n-2}{16(n-1)} \varepsilon^{n-2}\left(\varepsilon^{2}+|x|^{2}\right)^{2-n} \sum_{i, k, l=1}^{n}\left(\partial_{l} \bar{H}_{i k}(x)\right)^{2} .
$$

Using Corollary 18, we obtain

$$
\begin{aligned}
& \int_{\mathbb{R}^{n}} \varepsilon^{n-2}\left(\varepsilon^{2}+|x|^{2}\right)^{2-n} \sum_{i, k, l=1}^{n}\left(\partial_{l} \bar{H}_{i k}(x)\right)^{2} \\
& =\frac{1}{n}\left|S^{n-1}\right| \sum_{i, j, k, l=1}^{n}\left(W_{i j k l}+W_{i l k j}\right)^{2} \\
& \quad \cdot \int_{0}^{\infty}\left(1+r^{2}\right)^{2-n}\left[\varepsilon^{4} r^{n+1}-\frac{2(n+4)}{n+2} \varepsilon^{6} r^{n+3}+\frac{n+8}{n+2} \varepsilon^{8} r^{n+5}\right] d r .
\end{aligned}
$$

Moreover, we have

$$
\int_{0}^{\infty}\left(1+r^{2}\right)^{2-n} r^{n+1} d r=\frac{n-8}{n+2} \int_{0}^{\infty}\left(1+r^{2}\right)^{2-n} r^{n+3} d r
$$

and

$$
\int_{0}^{\infty}\left(1+r^{2}\right)^{2-n} r^{n+5} d r=\frac{n+4}{n-10} \int_{0}^{\infty}\left(1+r^{2}\right)^{2-n} r^{n+3} d r
$$

by Proposition 27. From this the assertion follows. 
Corollary 20. Assume that $n \geq 52$. Moreover, suppose that $\varepsilon_{*}>0$ is defined by

$$
\left(3+\sqrt{9-\frac{8(n+8)(n-8)}{(n+4)(n-10)}}\right) \varepsilon_{*}^{2}=\frac{2(n-8)}{n+4} .
$$

Then $\left(0, \varepsilon_{*}\right)$ is a critical point of the function $F(\xi, \varepsilon)$. Moreover, we have $\frac{\partial^{2}}{\partial \varepsilon^{2}} F\left(0, \varepsilon_{*}\right)$ $>0$.

In the next step, we show that $\left(0, \varepsilon_{*}\right)$ is a strict local minimum of the function $F$. To that end, we compute the Hessian of $F$ at a point $(0, \varepsilon)$.

Proposition 21. The second order partial derivatives of the function $F(\xi, \varepsilon)$ are given by

$$
\begin{aligned}
\frac{\partial^{2}}{\partial \xi_{p} \partial \xi_{q}} F(0, \varepsilon) & =\int_{\mathbb{R}^{n}}(n-2)^{2} \varepsilon^{n-2}\left(\varepsilon^{2}+|x|^{2}\right)^{-n} \sum_{l=1}^{n} \bar{H}_{p l}(x) \bar{H}_{q l}(x) \\
& -\int_{\mathbb{R}^{n}} \frac{(n-2)^{2}}{4} \varepsilon^{n-2}\left(\varepsilon^{2}+|x|^{2}\right)^{-n} \sum_{i, k, l=1}^{n}\left(\partial_{l} \bar{H}_{i k}(x)\right)^{2} x_{p} x_{q} \\
& +\int_{\mathbb{R}^{n}} \frac{(n-2)^{2}}{8(n-1)} \varepsilon^{n-2}\left(\varepsilon^{2}+|x|^{2}\right)^{1-n} \sum_{i, k, l=1}^{n}\left(\partial_{l} \bar{H}_{i k}(x)\right)^{2} \delta_{p q} .
\end{aligned}
$$

Proof. Using the identity

$$
\begin{aligned}
& \sum_{i, k, l=1}^{n} \bar{H}_{i l}(x) \bar{H}_{k l}(x) \partial_{i} u_{(\xi, \varepsilon)}(x) \partial_{k} u_{(\xi, \varepsilon)}(x) \\
& =(n-2)^{2} \varepsilon^{n-2}\left(\varepsilon^{2}+|x-\xi|^{2}\right)^{-n} \sum_{i, k, l=1}^{n} \bar{H}_{i l}(x) \bar{H}_{k l}(x)\left(x_{i}-\xi_{i}\right)\left(x_{k}-\xi_{k}\right) \\
& =(n-2)^{2} \varepsilon^{n-2}\left(\varepsilon^{2}+|x-\xi|^{2}\right)^{-n} \sum_{i, k, l=1}^{n} \bar{H}_{i l}(x) \bar{H}_{k l}(x) \xi_{i} \xi_{k},
\end{aligned}
$$

we obtain

$$
\begin{aligned}
& \left.\frac{\partial^{2}}{\partial \xi_{p} \partial \xi_{q}}\left(\frac{1}{2} \sum_{i, k, l=1}^{n} \bar{H}_{i l}(x) \bar{H}_{k l}(x) \partial_{i} u_{(\xi, \varepsilon)}(x) \partial_{k} u_{(\xi, \varepsilon)}(x)\right)\right|_{\xi=0} \\
& =(n-2)^{2} \varepsilon^{n-2}\left(\varepsilon^{2}+|x|^{2}\right)^{-n} \sum_{l=1}^{n} \bar{H}_{p l}(x) \bar{H}_{q l}(x) .
\end{aligned}
$$

Moreover, we have

$$
\begin{aligned}
& \left.\frac{\partial^{2}}{\partial \xi_{p} \partial \xi_{q}}\left(\frac{n-2}{16(n-1)} \sum_{i, k, l=1}^{n}\left(\partial_{l} \bar{H}_{i k}(x)\right)^{2} u_{(\xi, \varepsilon)}(x)^{2}\right)\right|_{\xi=0} \\
& =\frac{(n-2)^{2}}{4} \varepsilon^{n-2}\left(\varepsilon^{2}+|x|^{2}\right)^{-n} \sum_{i, k, l=1}^{n}\left(\partial_{l} \bar{H}_{i k}(x)\right)^{2} x_{p} x_{q} \\
& -\frac{(n-2)^{2}}{8(n-1)} \varepsilon^{n-2}\left(\varepsilon^{2}+|x|^{2}\right)^{1-n} \sum_{i, k, l=1}^{n}\left(\partial_{l} \bar{H}_{i k}(x)\right)^{2} \delta_{p q} .
\end{aligned}
$$


Finally, we have

$$
\begin{aligned}
& \sum_{i, k=1}^{n} \bar{H}_{i k}(x) \partial_{i} \partial_{k} u_{(\xi, \varepsilon)}(x) \\
& =n(n-2) \varepsilon^{\frac{n-2}{2}}\left(\varepsilon^{2}+|x-\xi|^{2}\right)^{-\frac{n+2}{2}} \sum_{i, k=1}^{n} \bar{H}_{i k}(x)\left(x_{i}-\xi_{i}\right)\left(x_{k}-\xi_{k}\right) \\
& =n(n-2) \varepsilon^{\frac{n-2}{2}}\left(\varepsilon^{2}+|x-\xi|^{2}\right)^{-\frac{n+2}{2}} \sum_{i, k=1}^{n} \bar{H}_{i k}(x) \xi_{i} \xi_{k}
\end{aligned}
$$

since $\bar{H}_{i k}(x)$ is trace-free. Thus, we conclude that

$$
\begin{aligned}
& \left.\frac{\partial^{2}}{\partial \xi_{p} \partial \xi_{q}}\left(\sum_{i, k=1}^{n} \bar{H}_{i k}(x) \partial_{i} \partial_{k} u_{(\xi, \varepsilon)}(x) z_{(\xi, \varepsilon)}(x)\right)\right|_{\xi=0} \\
& =2 n(n-2) \varepsilon^{\frac{n-2}{2}}\left(\varepsilon^{2}+|x|^{2}\right)^{-\frac{n+2}{2}} \sum_{i, k=1}^{n} \bar{H}_{p q}(x) z_{(0, \varepsilon)}(x)=0 .
\end{aligned}
$$

From this the assertion follows.

Proposition 22. The second order partial derivatives of the function $F(\xi, \varepsilon)$ are given by

$$
\begin{aligned}
& \frac{\partial^{2}}{\partial \xi_{p} \partial \xi_{q}} F(0, \varepsilon) \\
& =\frac{4(n-2)^{2}}{n(n+2)(n+4)}\left|S^{n-1}\right| \sum_{i, k, l=1}^{n}\left(W_{i p k l}+W_{i l k p}\right)\left(W_{i q k l}+W_{i l k q}\right) \\
& \cdot\left[\varepsilon^{4}-\frac{3(n+6)}{2(n-8)} \varepsilon^{6}\right] \int_{0}^{\infty}\left(1+r^{2}\right)^{-n} r^{n+5} d r \\
& +\frac{(n-2)^{2}}{n(n+2)(n+4)}\left|S^{n-1}\right| \sum_{i, j, k, l=1}^{n}\left(W_{i j k l}+W_{i l k j}\right)^{2} \delta_{p q} \\
& \cdot\left[\varepsilon^{4}-\frac{n+7}{n-8} \varepsilon^{6}\right] \int_{0}^{\infty}\left(1+r^{2}\right)^{-n} r^{n+5} d r .
\end{aligned}
$$

Proof. Using the identity

$$
\begin{aligned}
& \int_{\partial B_{r}(0)} \sum_{l=1}^{n} \bar{H}_{p l}(x) \bar{H}_{q l}(x) \\
& =\int_{\partial B_{r}(0)} \sum_{i, j, k, l, m=1}^{n} W_{i p k l} W_{j q m l} x_{i} x_{j} x_{k} x_{m}\left(1-|x|^{2}\right)^{2} \\
& =\frac{1}{n(n+2)}\left|S^{n-1}\right| \\
& \quad \cdot \sum_{i, j, k, l, m=1}^{n} W_{i p k l} W_{j q m l}\left(\delta_{i j} \delta_{k m}+\delta_{i k} \delta_{j m}+\delta_{i m} \delta_{j k}\right) r^{n+3}\left(1-r^{2}\right)^{2} \\
& =\frac{1}{2 n(n+2)}\left|S^{n-1}\right| \sum_{i, k, l=1}^{n}\left(W_{i p k l}+W_{i l k p}\right)\left(W_{i q k l}+W_{i l k q}\right) r^{n+3}\left(1-r^{2}\right)^{2},
\end{aligned}
$$


we obtain

$$
\begin{aligned}
& \int_{\mathbb{R}^{n}} \varepsilon^{n-2}\left(\varepsilon^{2}+|x|^{2}\right)^{-n} \sum_{i, k, l=1}^{n} \bar{H}_{p l}(x) \bar{H}_{q l}(x) \\
& =\frac{1}{2 n(n+2)}\left|S^{n-1}\right| \sum_{i, k, l=1}^{n}\left(W_{i p k l}+W_{i l k p}\right)\left(W_{i q k l}+W_{i l k q}\right) \\
& \cdot \int_{0}^{\infty}\left(1+r^{2}\right)^{-n}\left[\varepsilon^{2} r^{n+3}-2 \varepsilon^{4} r^{n+5}+\varepsilon^{6} r^{n+7}\right] d r .
\end{aligned}
$$

Similarly, it follows from Proposition 17 that

$$
\begin{aligned}
& \int_{\mathbb{R}^{n}} \varepsilon^{n-2}\left(\varepsilon^{2}+|x|^{2}\right)^{-n} \sum_{i, k, l=1}^{n}\left(\partial_{l} \bar{H}_{i k}(x)\right)^{2} x_{p} x_{q} \\
& =\frac{2}{n(n+2)}\left|S^{n-1}\right| \sum_{i, k, l=1}^{n}\left(W_{i p k l}+W_{i l k p}\right)\left(W_{i q k l}+W_{i l k q}\right) \\
& \cdot \int_{0}^{\infty}\left(1+r^{2}\right)^{-n}\left[\varepsilon^{2} r^{n+3}-\frac{2(n+8)}{n+4} \varepsilon^{4} r^{n+5}+\frac{n+16}{n+4} \varepsilon^{6} r^{n+7}\right] d r \\
& +\frac{1}{n(n+2)}\left|S^{n-1}\right| \sum_{i, j, k, l=1}^{n}\left(W_{i j k l}+W_{i l k j}\right)^{2} \delta_{p q} \\
& \cdot \int_{0}^{\infty}\left(1+r^{2}\right)^{-n}\left[\varepsilon^{2} r^{n+3}-\frac{2(n+6)}{n+4} \varepsilon^{4} r^{n+5}+\frac{n+10}{n+4} \varepsilon^{6} r^{n+7}\right] d r .
\end{aligned}
$$

Moreover, we have

$$
\begin{aligned}
& \int_{\mathbb{R}^{n}} \varepsilon^{n-2}\left(\varepsilon^{2}+|x|^{2}\right)^{1-n} \sum_{i, k, l=1}^{n}\left(\partial_{l} \bar{H}_{i k}(x)\right)^{2} \delta_{p q} \\
& =\frac{1}{n}\left|S^{n-1}\right| \sum_{i, j, k, l=1}^{n}\left(W_{i j k l}+W_{i l k j}\right)^{2} \delta_{p q} \\
& \quad \int_{0}^{\infty}\left(1+r^{2}\right)^{1-n}\left[\varepsilon^{2} r^{n+1}-\frac{2(n+4)}{n+2} \varepsilon^{4} r^{n+3}+\frac{n+8}{n+2} \varepsilon^{6} r^{n+5}\right] d r .
\end{aligned}
$$

by Corollary [18. Using Proposition 21 and the identity

$$
\int_{0}^{\infty}\left(1+r^{2}\right)^{1-n} r^{n+1} d r=\frac{2(n-1)}{n+2} \int_{0}^{\infty}\left(1+r^{2}\right)^{-n} r^{n+3} d r
$$


we obtain

$$
\begin{aligned}
& \frac{\partial^{2}}{\partial \xi_{p} \partial \xi_{q}} F(0, \varepsilon) \\
& =\frac{4(n-2)^{2}}{n(n+2)(n+4)}\left|S^{n-1}\right| \sum_{i, k, l=1}^{n}\left(W_{i p k l}+W_{i l k p}\right)\left(W_{i q k l}+W_{i l k q}\right) \\
& \cdot \int_{0}^{\infty}\left(1+r^{2}\right)^{-n}\left[\varepsilon^{4} r^{n+5}-\frac{3}{2} \varepsilon^{6} r^{n+7}\right] d r \\
& +\frac{(n-2)^{2}}{4 n(n+2)}\left|S^{n-1}\right| \sum_{i, j, k, l=1}^{n}\left(W_{i j k l}+W_{i l k j}\right)^{2} \delta_{p q} \\
& \cdot \int_{0}^{\infty}\left(1+r^{2}\right)^{-n}\left[\frac{2(n+6)}{n+4} \varepsilon^{4} r^{n+5}-\frac{n+10}{n+4} \varepsilon^{6} r^{n+7}\right] d r \\
& -\frac{(n-2)^{2}}{8 n(n-1)}\left|S^{n-1}\right| \sum_{i, j, k, l=1}^{n}\left(W_{i j k l}+W_{i l k j}\right)^{2} \delta_{p q} \\
& \cdot \int_{0}^{\infty}\left(1+r^{2}\right)^{1-n}\left[\frac{2(n+4)}{n+2} \varepsilon^{4} r^{n+3}-\frac{n+8}{n+2} \varepsilon^{6} r^{n+5}\right] d r .
\end{aligned}
$$

Hence, the assertion follows from the identities

$$
\begin{aligned}
& \int_{0}^{\infty}\left(1+r^{2}\right)^{-n} r^{n+7} d r=\frac{n+6}{n-8} \int_{0}^{\infty}\left(1+r^{2}\right)^{-n} r^{n+5} d r \\
& \int_{0}^{\infty}\left(1+r^{2}\right)^{1-n} r^{n+3} d r=\frac{2(n-1)}{n+4} \int_{0}^{\infty}\left(1+r^{2}\right)^{-n} r^{n+5} d r \\
& \int_{0}^{\infty}\left(1+r^{2}\right)^{1-n} r^{n+5} d r=\frac{2(n-1)}{n-8} \int_{0}^{\infty}\left(1+r^{2}\right)^{-n} r^{n+5} d r
\end{aligned}
$$

Corollary 23. Assume that $n \geq 52$ and $\varepsilon_{*}>0$ is defined by (6). Then the function $F(\xi, \varepsilon)$ has a strict local minimum at the point $\left(0, \varepsilon_{*}\right)$.

Proof. It follows from Corollary 20 that $\left(0, \varepsilon_{*}\right)$ is a critical point of the function $F(\xi, \varepsilon)$. Moreover, we have $\frac{\partial^{2}}{\partial \varepsilon^{2}} F\left(0, \varepsilon_{*}\right)>0$. Since $n \geq 52$, we have

$$
\frac{6}{n+4}<\sqrt{9-\frac{8(n+8)(n-8)}{(n+4)(n-10)}} \text {. }
$$

This implies

$$
\frac{3(n+6)}{n+4} \varepsilon_{*}^{2}<\left(3+\sqrt{9-\frac{8(n+8)(n-8)}{(n+4)(n-10)}}\right) \varepsilon_{*}^{2}=\frac{2(n-8)}{n+4} .
$$

Thus, we conclude that

$$
\frac{n+7}{n-8} \varepsilon_{*}^{2}<\frac{3(n+6)}{2(n-8)} \varepsilon_{*}^{2}<1
$$

Hence, it follows from Proposition 22 that the matrix $\frac{\partial^{2}}{\partial \xi_{p} \partial \xi_{q}} F\left(0, \varepsilon_{*}\right)$ is positive definite. This proves the assertion. 


\section{Proof of the MAIN THEOREM}

Proposition 24. Assume that $n \geq 52$. Moreover, let $g$ be a smooth metric on $\mathbb{R}^{n}$ of the form $g(x)=\exp (h(x))$, where $h(x)$ is a trace-free symmetric two-tensor on $\mathbb{R}^{n}$ such that $|h(x)|+|\partial h(x)|+\left|\partial^{2} h(x)\right| \leq \alpha \leq \alpha_{1}$ for all $x \in \mathbb{R}^{n}, h(x)=0$ for $|x| \geq 1$, and $h_{i k}(x)=\mu\left(\lambda^{2}-|x|^{2}\right) H_{i k}(x)$ for $|x| \leq \rho$. As above, we assume that $\lambda \leq \rho \leq 1$ and $\mu \leq 1$. If $\alpha$ and $\rho^{2-n} \mu^{-2} \lambda^{n-10}$ are sufficiently small, then there exists a positive function $v$ such that

$$
\begin{gathered}
\Delta_{g} v-\frac{n-2}{4(n-1)} R_{g} v+n(n-2) v^{\frac{n+2}{n-2}}=0, \\
\int_{\mathbb{R}^{n}} v^{\frac{2 n}{n-2}}<\left(\frac{Y\left(S^{n}\right)}{4 n(n-1)}\right)^{\frac{n}{2}},
\end{gathered}
$$

and $\sup _{|x| \leq \lambda} v(x) \geq c \lambda^{\frac{2-n}{2}}$. Here, $c$ is a positive constant that depends only on $n$.

Proof. By Corollary 23, the function $F(\xi, \varepsilon)$ has a strict local minimum at $\left(0, \varepsilon_{*}\right)$. Hence, we can find an open set $\Omega^{\prime} \subset \Omega$ such that $\left(0, \varepsilon_{*}\right) \in \Omega^{\prime}$ and

$$
F\left(0, \varepsilon_{*}\right)<\inf _{(\xi, \varepsilon) \in \partial \Omega^{\prime}} F(\xi, \varepsilon)<0 .
$$

Using Corollary 14, we obtain

$$
\begin{aligned}
& \left|\mathcal{F}_{g}(\lambda \xi, \lambda \varepsilon)-\lambda^{8} \mu^{2} F(\xi, \varepsilon)\right| \\
& \leq C \lambda^{\frac{8 n}{n-2}} \mu^{\frac{2 n}{n-2}}+C \lambda^{4} \mu\left(\frac{\lambda}{\rho}\right)^{\frac{n-2}{2}}+C\left(\frac{\lambda}{\rho}\right)^{n-2}
\end{aligned}
$$

for all $(\xi, \varepsilon) \in \Omega$. This implies

$$
\begin{aligned}
& \left|\lambda^{-8} \mu^{-2} \mathcal{F}_{g}(\lambda \xi, \lambda \varepsilon)-F(\xi, \varepsilon)\right| \\
& \leq C \lambda^{\frac{16}{n-2}} \mu^{\frac{4}{n-2}}+C \rho^{\frac{2-n}{2}} \mu^{-1} \lambda^{\frac{n-10}{2}}+C \rho^{2-n} \mu^{-2} \lambda^{n-10}
\end{aligned}
$$

for all $(\xi, \varepsilon) \in \Omega$. Hence, if $\rho^{2-n} \mu^{-2} \lambda^{n-10}$ is sufficiently small, then we have

$$
\mathcal{F}_{g}\left(0, \lambda \varepsilon_{*}\right)<\inf _{(\xi, \varepsilon) \in \partial \Omega^{\prime}} \mathcal{F}_{g}(\lambda \xi, \lambda \varepsilon)<0 .
$$

Consequently, there exists a point $(\bar{\xi}, \bar{\varepsilon}) \in \Omega^{\prime}$ such that

$$
\mathcal{F}_{g}(\lambda \bar{\xi}, \lambda \bar{\varepsilon})=\inf _{(\xi, \varepsilon) \in \Omega^{\prime}} \mathcal{F}_{g}(\lambda \xi, \lambda \varepsilon)<0
$$

By Proposition [6 the function $v=v_{(\lambda \bar{\xi}, \lambda \bar{\varepsilon})}$ is a non-negative weak solution of the partial differential equation

$$
\Delta_{g} v-\frac{n-2}{4(n-1)} R_{g} v+n(n-2) v^{\frac{n+2}{n-2}}=0 .
$$

Using a result of $\mathrm{N}$. Trudinger, we conclude that $v$ is smooth (see [20], Theorem 3 on p. 271). Moreover, we have

$$
\begin{aligned}
2(n-2) \int_{\mathbb{R}^{n}} v^{\frac{2 n}{n-2}} & =2(n-2)\left(\frac{Y\left(S^{n}\right)}{4 n(n-1)}\right)^{\frac{n}{2}}+\mathcal{F}_{g}(\lambda \bar{\xi}, \lambda \bar{\varepsilon}) \\
& <2(n-2)\left(\frac{Y\left(S^{n}\right)}{4 n(n-1)}\right)^{\frac{n}{2}} .
\end{aligned}
$$


Finally, it follows from Proposition 5 that $\left\|v-u_{(\lambda \bar{\xi}, \lambda \bar{\varepsilon})}\right\|_{L^{\frac{2 n}{n-2}}\left(\mathbb{R}^{n}\right)} \leq C \alpha$. This implies

$$
\left|B_{\lambda}(0)\right|^{\frac{n-2}{2 n}} \sup _{|x| \leq \lambda} v(x) \geq\|v\|_{L^{\frac{2 n}{n-2}}\left(B_{\lambda}(0)\right)} \geq\left\|u_{(\lambda \bar{\xi}, \lambda \bar{\varepsilon})}\right\|_{L^{\frac{2 n}{n-2}}\left(B_{\lambda}(0)\right)}-C \alpha .
$$

Hence, if $\alpha$ is sufficiently small, then we obtain $\lambda^{\frac{n-2}{2}} \sup _{|x| \leq \lambda} v(x) \geq c$.

Proposition 25. Let $n \geq 52$. Then there exists a smooth metric $g$ on $\mathbb{R}^{n}$ with the following properties:

(i) $g_{i k}(x)=\delta_{i k}$ for $|x| \geq \frac{1}{2}$,

(ii) $g$ is not conformally flat,

(iii) there exists a sequence of non-negative smooth functions $v_{\nu}(\nu \in \mathbb{N})$ such that

$$
\Delta_{g} v_{\nu}-\frac{n-2}{4(n-1)} R_{g} v_{\nu}+n(n-2) v_{\nu}^{\frac{n+2}{n-2}}=0
$$

for all $\nu \in \mathbb{N}$,

$$
\int_{\mathbb{R}^{n}} v_{\nu}^{\frac{2 n}{n-2}}<\left(\frac{Y\left(S^{n}\right)}{4 n(n-1)}\right)^{\frac{n}{2}}
$$

for all $\nu \in \mathbb{N}$, and $\sup _{|x| \leq 1} v_{\nu}(x) \rightarrow \infty$ as $\nu \rightarrow \infty$.

Proof. Choose a smooth cutoff function $\eta: \mathbb{R} \rightarrow \mathbb{R}$ such that $\eta(t)=1$ for $t \leq 1$ and $\eta(t)=0$ for $t \geq 2$. We define a trace-free symmetric two-tensor on $\mathbb{R}^{n}$ by

$$
h_{i k}(x)=\sum_{N=N_{0}}^{\infty} \eta\left(4 N^{2}\left|x-y_{N}\right|\right) 2^{-N}\left(2^{-N}-\left|x-y_{N}\right|^{2}\right) H_{i k}\left(x-y_{N}\right),
$$

where $y_{N}=\left(\frac{1}{N}, 0, \ldots, 0\right) \in \mathbb{R}^{n}$. It is straightforward to verify that $h(x)$ is $C^{\infty}$ smooth.

Let $\alpha$ be the constant appearing in Proposition 24. If $N_{0}$ is sufficiently large, then we have $|h(x)|+|\partial h(x)|+\left|\partial^{2} h(x)\right| \leq \alpha$ for all $x \in \mathbb{R}^{n}$ and $h(x)=0$ for $|x| \geq \frac{1}{2}$. Moreover, we have $h_{i k}(x)=2^{-N}\left(2^{-N}-\left|x-y_{N}\right|^{2}\right) H_{i k}\left(x-y_{N}\right)$ provided that $N \geq N_{0}$ and $\left|x-y_{N}\right| \leq \frac{1}{4 N^{2}}$. Hence, we can apply Proposition 24 with $\lambda=2^{-N / 2}, \mu=2^{-N}$, and $\rho=\frac{1}{4 N^{2}}$. From this the assertion follows.

\section{Appendix A. An ASYmptotic EXPANSION for the SCALAR CURVATURE}

Suppose that $h(x)$ is a trace-free symmetric two-tensor defined on $\mathbb{R}^{n}$ satisfying $|h(x)| \leq 1$ for all $x \in \mathbb{R}^{n}$. We define a Riemannian metric $g$ on $\mathbb{R}^{n}$ by $g(x)=\exp (h(x))$. In this section, we derive an approximate formula for the scalar curvature of this metric. A similar formula is derived in [2].

Proposition 26. Let $R_{g}$ be the scalar curvature of $g$. There exists a constant $C$, depending only on $n$, such that

$$
\begin{aligned}
& \left|R_{g}-\partial_{i} \partial_{k} h_{i k}+\partial_{i}\left(h_{i l} \partial_{k} h_{k l}\right)-\frac{1}{2} \partial_{i} h_{i l} \partial_{k} h_{k l}+\frac{1}{4} \partial_{l} h_{i k} \partial_{l} h_{i k}\right| \\
& \leq C|h|^{2}\left|\partial^{2} h\right|+C|h||\partial h|^{2} .
\end{aligned}
$$

Proof. The Riemann curvature tensor is defined as

$$
\partial_{i} \Gamma_{j k}^{m}-\partial_{j} \Gamma_{i k}^{m}+\Gamma_{j k}^{l} \Gamma_{i l}^{m}-\Gamma_{i k}^{l} \Gamma_{j l}^{m}
$$


Hence, the scalar curvature of $g$ is given by

$$
R_{g}=g^{j k}\left(\partial_{i} \Gamma_{j k}^{i}-\partial_{j} \Gamma_{i k}^{i}+\Gamma_{j k}^{l} \Gamma_{i l}^{i}-\Gamma_{i k}^{l} \Gamma_{j l}^{i}\right)
$$

Since $h$ is trace-free, we have $\operatorname{det} g(x)=1$ for all $x \in \mathbb{R}^{n}$. This implies $\Gamma_{i k}^{i}=$ $\frac{1}{2} g^{i l} \partial_{k} g_{i l}=\frac{1}{2} \partial_{k} \log \operatorname{det} g=0$. Therefore, we obtain

$$
\begin{aligned}
R_{g} & =g^{j k} \partial_{i} \Gamma_{j k}^{i}-g^{j k} \Gamma_{i k}^{l} \Gamma_{j l}^{i} \\
& =\partial_{i}\left(g^{j k} \Gamma_{j k}^{i}\right)+g^{j k} \Gamma_{i k}^{l} \Gamma_{j l}^{i} .
\end{aligned}
$$

Note that

From this it follows that

$$
g^{j k} \Gamma_{j k}^{i}=g^{i l} g^{j k} \partial_{k} g_{j l} .
$$

$$
\begin{aligned}
& \left|\partial_{i}\left(g^{j k} \Gamma_{j k}^{i}\right)-\partial_{i} \partial_{k} h_{i k}+\frac{1}{2} \partial_{i}\left(h_{i l} \partial_{k} h_{k l}\right)+\frac{1}{2} \partial_{i}\left(h_{k l} \partial_{k} h_{i l}\right)\right| \\
& \leq C|h|^{2}\left|\partial^{2} h\right|+C|h||\partial h|^{2}
\end{aligned}
$$

hence

$$
\begin{aligned}
& \left|\partial_{i}\left(g^{j k} \Gamma_{j k}^{i}\right)-\partial_{i} \partial_{k} h_{i k}+\partial_{i}\left(h_{i l} \partial_{k} h_{k l}\right)-\frac{1}{2} \partial_{i} h_{i l} \partial_{k} h_{k l}+\frac{1}{2} \partial_{i} h_{k l} \partial_{k} h_{i l}\right| \\
& \leq C|h|^{2}\left|\partial^{2} h\right|+C|h||\partial h|^{2} .
\end{aligned}
$$

Moreover, we have

$$
\left|g^{j k} \Gamma_{i k}^{l} \Gamma_{j l}^{i}+\frac{1}{4} \partial_{l} h_{i k} \partial_{l} h_{i k}-\frac{1}{2} \partial_{i} h_{k l} \partial_{k} h_{i l}\right| \leq C|h||\partial h|^{2} .
$$

Putting these facts together, we obtain

$$
\begin{aligned}
& \left|R_{g}-\partial_{i} \partial_{k} h_{i k}+\partial_{i}\left(h_{i l} \partial_{k} h_{k l}\right)-\frac{1}{2} \partial_{i} h_{i l} \partial_{k} h_{k l}+\frac{1}{4} \partial_{l} h_{i k} \partial_{l} h_{i k}\right| \\
& \leq C|h|^{2}\left|\partial^{2} h\right|+C|h||\partial h|^{2} .
\end{aligned}
$$

This completes the proof.

\section{Appendix B. Some useful identities}

Proposition 27. Suppose that $\alpha$ and $\beta$ are real numbers satisfying $2 \alpha-2>\beta+1>$ 0. Then

$$
\int_{0}^{\infty}\left(1+r^{2}\right)^{1-\alpha} r^{\beta} d r=\frac{2 \alpha-2}{2 \alpha-\beta-3} \int_{0}^{\infty}\left(1+r^{2}\right)^{-\alpha} r^{\beta} d r
$$

and

$$
\int_{0}^{\infty}\left(1+r^{2}\right)^{-\alpha} r^{\beta+2} d r=\frac{\beta+1}{2 \alpha-\beta-3} \int_{0}^{\infty}\left(1+r^{2}\right)^{-\alpha} r^{\beta} d r
$$

Proof. Using the fundamental theorem of calculus, we obtain

$$
\begin{aligned}
0 & =\int_{0}^{\infty} \frac{d}{d r}\left[\left(1+r^{2}\right)^{1-\alpha} r^{\beta+1}\right] d r \\
& =(\beta+1) \int_{0}^{\infty}\left(1+r^{2}\right)^{1-\alpha} r^{\beta} d r-(2 \alpha-2) \int_{0}^{\infty}\left(1+r^{2}\right)^{-\alpha} r^{\beta+2} d r
\end{aligned}
$$

From this the assertion follows.

Proposition 28. Suppose that $p(x)$ is a homogenous polynomial of degree $d$. Then

$$
\int_{\partial B_{1}(0)} p(x)=\frac{1}{d(n+d-2)} \int_{\partial B_{1}(0)} \Delta p(x) .
$$


Proof. Using the divergence theorem, we obtain

$$
\begin{aligned}
\int_{\partial B_{1}(0)} \Delta p(x) & =(n+d-2) \int_{B_{1}(0)} \Delta p(x) \\
& =(n+d-2) \int_{\partial B_{1}(0)} \sum_{k=1}^{n} x_{k} \partial_{k} p(x) \\
& =d(n+d-2) \int_{\partial B_{1}(0)} p(x) .
\end{aligned}
$$

Corollary 29. We have

$$
\begin{gathered}
\int_{\partial B_{1}(0)} x_{i} x_{j}=\frac{1}{n}\left|S^{n-1}\right| \delta_{i j} \\
\int_{\partial B_{1}(0)} x_{i} x_{j} x_{k} x_{l}=\frac{1}{n(n+2)}\left|S^{n-1}\right|\left(\delta_{i j} \delta_{k l}+\delta_{i k} \delta_{j l}+\delta_{i l} \delta_{j k}\right),
\end{gathered}
$$

and

$$
\begin{aligned}
& \int_{\partial B_{1}(0)} x_{i} x_{j} x_{k} x_{l} x_{p} x_{q} \\
& =\frac{1}{n(n+2)(n+4)}\left|S^{n-1}\right|\left(\delta_{i j} \delta_{k l} \delta_{p q}+\delta_{i j} \delta_{k p} \delta_{l q}+\delta_{i j} \delta_{k q} \delta_{l p}\right. \\
& +\delta_{i k} \delta_{j l} \delta_{p q}+\delta_{i k} \delta_{j p} \delta_{l q}+\delta_{i k} \delta_{j q} \delta_{l p} \\
& +\delta_{i l} \delta_{j k} \delta_{p q}+\delta_{i l} \delta_{j p} \delta_{k q}+\delta_{i l} \delta_{j q} \delta_{k p} \\
& +\delta_{i p} \delta_{j k} \delta_{l q}+\delta_{i p} \delta_{j l} \delta_{k q}+\delta_{i p} \delta_{j q} \delta_{k l} \\
& \left.+\delta_{i q} \delta_{j k} \delta_{l p}+\delta_{i q} \delta_{j l} \delta_{k p}+\delta_{i q} \delta_{j p} \delta_{k l}\right) \text {. }
\end{aligned}
$$

\section{ACKNOWLEDGMENTS}

It is a pleasure to thank Professor Richard Schoen and Professor Fernando Marques for numerous discussions on this subject.

\section{REFERENCES}

[1] A. Ambrosetti, Multiplicity results for the Yamabe problem on $S^{n}$, Proc. Natl. Acad. Sci. USA 99 (2002), 15252-15256. MR.1946759 (2003j:53047)

[2] A. Ambrosetti and A. Malchiodi, A multiplicity result for the Yamabe problem on $S^{n}$, J. Funct. Anal. 168, 529-561 (1999). MR1719213 (2000k:53032)

[3] T. Aubin, Équations différentielles non linéaires et problème de Yamabe concernant la courbure scalaire, J. Math. Pures Appl. 55, 269-296 (1976). MR0431287 (55:4288)

[4] T. Aubin, Sur quelques problèmes de courbure scalaire, J. Funct. Anal. 240, 269-289 (2006). MR2259897

[5] T. Aubin, Solution complète de la $C^{0}$ compacité de l'ensemble des solutions de l'équation de Yamabe, J. Funct. Anal. 244, 579-589 (2007). MR2297036

[6] M. Berti and A. Malchiodi, Non-compactness and multiplicity results for the Yamabe problem on $S^{n}$, J. Funct. Anal. 180, 210-241 (2001). MR1814428 (2002b:53049)

[7] O. Druet, Compactness for Yamabe metrics in low dimensions, Internat. Math. Res. Notices 23, 1143-1191 (2004). MR2041549(2005b:53056)

[8] O. Druet and E. Hebey, Blow-up examples for second order elliptic PDEs of critical Sobolev growth, Trans. Amer. Math. Soc. 357, 1915-1929 (2004). MR2115082 (2005i:58023)

[9] O. Druet and E. Hebey, Elliptic equations of Yamabe type, International Mathematics Research Surveys 1, 1-113 (2005). MR2148873(2006b:53046) 
[10] M. Khuri, F. Marques, and R. Schoen, A compactness theorem for the Yamabe problem, preprint (2007).

[11] Y.Y. Li and L. Zhang, Compactness of solutions to the Yamabe problem II, Calc. Var. PDE 24, 185-237 (2005). MR2164927 (2006f:53049)

[12] Y.Y. Li and M. Zhu, Yamabe type equations on three-dimensional Riemannian manifolds, Commun. Contemp. Math. 1, 1-50 (1999). MR1681811(2000m:53051)

[13] F.C. Marques, A-priori estimates for the Yamabe problem in the non-locally conformally flat case, J. Diff. Geom. 71, 315-346 (2005). MR2197144 (2006i:53046)

[14] D. Pollack, Nonuniqueness and high energy solutions for a conformally invariant scalar equation, Comm. Anal. Geom. 1, 347-414 (1993). MR.1266473(94m:58051)

[15] O. Rey, The role of the Green's function in a non-linear elliptic equation involving the critical Sobolev exponent, J. Funct. Anal. 89, 1-52 (1990). MR1040954 (91b:35012)

[16] R.M. Schoen, Conformal deformation of a Riemannian metric to constant scalar curvature, J. Diff. Geom. 20, 479-495 (1984) MR.788292 (86i:58137)

[17] R.M. Schoen, Variational theory for the total scalar curvature functional for Riemannian metrics and related topics, Topics in the calculus of variations (ed. by Mariano Giaquinta), Lecture Notes in Mathematics, vol. 1365, Springer Verlag, 1989, 120-154. MR994021 (90g:58023)

[18] R.M. Schoen, On the number of constant scalar curvature metrics in a conformal class, Differential geometry (ed. by H. Blaine Lawson, Jr., and Keti Tenenblat), Pitman Monographs and Surveys in Pure and Applied Mathematics, vol. 52, Longman Scientific \& Technical, 1991, 311-320. MR1173050 (94e:53035)

[19] R.M. Schoen, A report on some recent progress on nonlinear problems in geometry, In: Surveys in differential geometry, Lehigh University, Bethlehem, PA, 1991, 201-241. MR1144528 (92m:53069)

[20] N. Trudinger, Remarks concerning the conformal deformation of Riemannian structures on compact manifolds, Annali Scuola Norm. Sup. Pisa 22, 265-274 (1968). MR0240748(39:2093)

Department of Mathematics, Stanford University, Stanford, California 94305 